\title{
A Novel Role for Nucleolin in Splice Site Selection
}

Kinneret Shefer ${ }^{1, \#}$, Ayub Boulos ${ }^{1, \#}$, Valer Gotea ${ }^{2, \#}$, Yair Ben Chaim ${ }^{3}$, Joseph Sperling ${ }^{4}$, Laura Elnitski ${ }^{2, *}$ and Ruth Sperling ${ }^{1, *}$

${ }^{1}$ Department of Genetics, The Hebrew University of Jerusalem, Jerusalem 91904, Israel

${ }^{2}$ Translational and Functional Genomics Branch, National Human Genome Research Institute, NIH,

Bethesda, MD 20892, USA

${ }^{3}$ Department of Natural Sciences, The Open University, Raanana 43107, Israel

${ }^{4}$ Department of Organic Chemistry, The Weizmann Institute of Science, Rehovot 76100, Israel

\# The first 3 authors should be regarded as joint First Authors

${ }^{*}$ To whom correspondence should be addressed.

Ruth Sperling

Department of Genetics

The Hebrew University of Jerusalem, Jerusalem 91904, Israel

Tel: +972-54-882-0311

Fax: +972-2-658-6975

Email: r.sperling@mail.huji.ac.il

Correspondence may also be addressed to:

Laura Elnitski

Translational and Functional Genomics Branch

National Human Genome Research Institute, NIH, Bethesda, MD 20892, USA

Tel: +1-301-451-0265

Fax: +1-301-402-4929

Email: elnitski@mail.nih.gov

We would like to dedicate this manuscript to the memory of Joseph (Yossi) Sperling.

Running Title: A Novel Role for Nucleolin in Splicing

\section{Keywords}

5' splice site selection, suppression of splicing, alternative splicing, endogenous spliceosome, latent splice sites, latent splicing, splicing regulation, mass spectrometry, RNA sequencing, bioinformatics analysis.

\section{This PDF file includes:}

Main Text

Figures 1 to 7

Table 1

Supplementary Information 


\begin{abstract}
Latent 5 ' splice sites are highly abundant in human introns, yet, are apparently not normally used. Splicing at most of these sites would incorporate in-frame stop codons generating nonsense mRNAs. Importantly, under stress and in cancer, splicing at latent sites is activated generating nonsense mRNAs from thousands of genes. Previous studies point to an unresolved RNA quality control mechanism that suppresses latent splicing independently of NMD. They further demonstrated a pivotal role for initiator-tRNA in this mechanism, through its interaction with the AUG codon, independent of its role in protein translation. To further elucidate this mechanism, here we searched for nuclear proteins directly bound to initiator-tRNA in the nucleus. We identified nucleolin (NCL), a multifunctional, abundant, and conserved protein, as a novel regulator of splice site selection. Starting with UV crosslinking, we show that NCL is directly and specifically interacting with initiator-tRNA in the nucleus, but not in the cytoplasm. In support of NCL involvement in this mechanism, we show activation of latent splicing in hundreds of transcripts upon NCL knockdown, disrupting gene transcripts involved in several important cellular pathways and cell metabolism functions (e.g. transcription factors, oncogenes, kinases, splicing factors, translation factors, and genes affecting cell motility, proliferation, and cellular trafficking). We thus propose NCL, a component of the endogenous spliceosome, through its direct interaction with initiator-tRNA and its effect on latent splicing as the first documented protein of a nuclear quality control mechanism that regulates splice site selection to protect cells from latent splicing that would generate defective mRNAs.
\end{abstract}




\section{Introduction}

All multi-exon genes undergo regulated splicing, and most are subject to alternative splicing (AS), which provides a major source of diversity in the human proteome and contributes largely to regulation of gene expression (reviewed in refs. 1-6). Importantly, misregulation of splicing, through mutations or level changes of either regulatory signals or spliceosome components and splicing regulatory proteins, contributes to several human diseases, including cancer (7-10).

A key step in constitutive and alternative pre-mRNA splicing is recognition and selection of a consensus sequence that defines the 5' splice site (5'SS). The 5'SS consensus sequence, AG/GTRAGT (where R denotes purine and "” denotes the splice junction), is well defined and remarkably similar among many organisms, from mammals to plants (11-13). Surprisingly, surveys of human gene annotations demonstrated that intronic sequences abound in 5'SS consensus sequences that are not involved in splicing under normal conditions (termed latent 5'SS), exceeding the number of authentic 5'SSs by a factor of 6 to 9 -fold $(14,15)$. Notably, the intronic sequences upstream of almost all $(>98 \%)$ of these sites harbor at least one in-frame stop codon, and therefore have the potential to introduce premature termination codons (PTCs) into the alternatively spliced isoforms (15). mRNAs that contain PTCs are nonsense mRNAs that can be harmful to cells as their truncated proteins are likely non-functional and can have potentially deleterious dominant effects on the cell's metabolism (16). Although splicing at latent sites has not been observed under normal growth, latent 5'SSs have been shown to be legitimate and can be activated. Latent splicing has been previously elicited in three different ways: (i) by eliminating the stop codons (in several gene constructs) either by point mutations that converted the stop codons to sense codons or by indels that shift the reading frame upstream of the stop codons $(17,18)$; (ii) by disrupting the reading frame through mutating the start ATG codon $(19,20)$; or (iii) by subjecting cells to stress conditions, such as heat shock or in cancer $(15,18$, $19,21,22)$.

Two scenarios can account for why splicing at latent sites have not been observed under normal growth: (i) splicing at latent 5'SSs does occur, but an RNA surveillance mechanism, such as NMD (23-25), rapidly and efficiently degrades the nonsense mRNAs to a level below detection; or (ii) there is an unknown suppression mechanism of splicing at latent 5 'SSs that are preceded by at least one stop codon in-frame with the upstream exon. Experiments have ruled out the first scenario of NMD (17-19, 22), or degradation by a yet unknown RNA degradation mechanism (20), while fitting the second scenario of latent splicing suppression. Latent splicing was shown to be regulated through the maintenance of an open reading frame $(17-20,22)$ and can be up-regulated under stress conditions $(15,21,22)$. These discoveries suggest the existence of an RNA quality control mechanism - termed suppression of splicing (SOS) - whose proposed function is to suppress the use of latent 5'SSs that would generate nonsense RNA transcripts (26, 27). Support for nuclear recognition of a PTC-harboring pre-mRNA and suppression of splicing to prevent such transcripts has been observed in a number of studies (28-30), including a study that showed nuclear retention of unspliced PTC-harboring transcripts at their genomic loci (31). However, the mechanism of SOS quality control remains nebulous.

Previous experiments showed that SOS regulation requires an open reading frame $(17,18)$ and an initiation codon $(19,20)$, suggesting a role for the initiator-tRNA (ini-tRNA) in this regulation (20). Indeed, ini-tRNA, which was found associated with the endogenous spliceosome, was found to act as a pre-mRNA splicing regulator, independent of its role in translation, and was identified as a potential SOS factor (20). Mutations in the AUG translation initiation codon led to activation of latent splicing, but could be compensated for by expressing ini-tRNA constructs carrying complementary anticodon mutations, which suppressed latent splicing. This effect was specific to ini-tRNA, as elongator-tRNA, whether having complementary mutation to the mutated AUG or not, did not rescue SOS (20). Thus, ini-tRNA that recognizes the AUG sequence through base pairing is a pivotal element in the predicted SOS mechanism. Its interaction with the AUG sequence, probably in a complex with auxiliary proteins, would establish a register for the recognition of the reading frame required for $\operatorname{SOS}(20,27,32)$, which otherwise would not be discernible in the nucleus. Ini-tRNA presumably functions at the initial steps of SOS, conveying SOS components to the pre-mRNA.

Building on the previous discovery that ini-tRNA represents a key element in SOS, here we search for cellular components that directly interact with ini-tRNA in the nucleus to help decipher the SOS mechanism. We identify nucleolin (NCL), an abundant, highly conserved and multifunctional protein, which we previously reported as being associated with the endogenous spliceosome (33), as a new SOS factor. We show that NCL is directly and specifically interacting with ini-tRNA in the nucleus, but not in 
the cytoplasm. Furthermore, when we knocked down NCL we observed activation of latent splicing at hundreds of latent 5'SSs that introduce in-frame STOP codons, disrupting gene transcripts involved in several important cellular pathways and cell metabolism functions. These results suggest a novel role for NCL in splice site selection as a component of the SOS quality control mechanism proposed to protect cells from the use of latent 5'SSs that would generate mRNA molecules with PTCs.

\section{Results}

\section{Search for Proteins Directly Associated with ini-tRNA in the Nucleus}

To further explore the mechanism of SOS, we searched for factors directly associated with ini-tRNA only in the nucleus but not in the cytoplasm. In our first round of experiments, we injected ${ }^{32} \mathrm{P}$-labeled ini-tRNA into either the nucleus or cytoplasm of Xenopus laevis (Xenopus) oocytes, followed by UV crosslinking and RNase digestion (Figure 1A; see Materials and Methods for details). We found ini-tRNA directly associated with specific proteins in the nuclei as represented by two radioactive bands of apparent MW of $\sim 60$ and $\sim 120 \mathrm{kDa}$ (Figure 1B; lanes 5-8). Samples not treated by RNase revealed two major bands of apparent MW of $120 \mathrm{kDA}$ and $170 \mathrm{kDA}$ (see Figure 1D). The 60 and $120 \mathrm{kDa}$ bands were not found in the cytoplasmic fraction samples (which only contained a $\sim 26 \mathrm{kDa}$ band) (Figure 1B, lane 1) or when the sample was not exposed to UV radiation (Figure 1B, lane 3).

Importantly, this association of ini-tRNA with the factors represented by the 60 and $120 \mathrm{kDA}$ bands is specific, because these bands, which only appeared after UV crosslinking and were not visible without it (Figure 1C, lanes 2 and 1, respectively), were chased by the addition of a hundred-fold cold ini-tRNA (Figure 1C, lane 3). Yet, they were hardly affected by a 100-fold excess cold elongator-tRNA (Figure 1C, lane 4). It should be pointed out that elongator-tRNA is a suitable negative control, because it is not associated with the endogenous spliceosome and it does not play a role in SOS (20). Specifically, activation of latent splicing induced by mutations in the translation initiation AUG codon, were suppressed by initRNA constructs carrying anticodon mutations that compensate for the AUG mutations. In contrast, elongator-tRNA, either WT or mutated to complement the mutated AUG did not suppress activation of latent splicing, as demonstrated for three different transcripts (20). These observations indicate that factors with apparent molecular weight of 60 and $120 \mathrm{kDa}$ were directly and specifically bound to ini-tRNA in the nucleus, but not in the cytoplasm.

To affinity purify the proteins associated with ini-tRNA in the nucleus, the experiment (see Figure 1A) was repeated, this time by injecting biotinylated ini-tRNA into Xenopus oocytes nuclei. Next, biotinylated ini-tRNAs with crosslinked proteins were affinity purified using streptavidin magnetic beads. As can be seen in Figure 1D, SDS PAGE of affinity-purified ini-tRNA with crosslinked components revealed two bands of associated components with apparent MW of 120 and $170 \mathrm{kDa}$, which yielded two respective bands of apparent MW of 60 and $120 \mathrm{kDa}$ after RNase digestion (Figure 1D, lane 1, left and right, respectively). These two bands have the same apparent MW as the bands obtained previously, i.e., without affinity purification. The control (affinity-purified non-biotinylated ${ }^{32} \mathrm{P}$-labeled ini-tRNA) did not yield any product (Figure 1D, lane 2, left and right).

To increase the yield of affinity-purified peptides, we repeated the experiment, this time replacing injection by incubation of biotinylated ${ }^{32} \mathrm{P}$-labeled ini-tRNA with an extract from one isolated Xenopus nucleus or cytoplasm. Again, we obtained two bands of apparent MW of 170 and $120 \mathrm{kDa}$ observed only in the nuclear extract and not in the cytoplasmic extract (Figure 2A, lanes 1 and 3, respectively) nor in the control (Figure 2A, lane 2). When we incubated our nuclear Xenopus extracts with increasing quantities $\left(0.5-2\right.$ picomol) of biotinylated ${ }^{32} \mathrm{P}-$-labeled ini-tRNA, the RNase treated products yielded 60 and $120 \mathrm{kDa}$ bands again (Figure 2B, lanes 1-3). The intensity of the obtained bands increased concomitantly with increasing quantities of ini-tRNA. No bands were observed when the experiment was repeated with the cytoplasmic extract (Figure 2B, lanes 4-6), even with increased levels of ini-tRNA. Thus, the use of nuclear extract in our experiments enabled us to increase the amount of ini-tRNA from $10 \mathrm{fmol} /$ nucleus to 2 picomol/nucleus in the procedure. These experiments show the direct and specific association between initRNA and nuclear components having apparent MW of $60 \mathrm{kDa}$ and $120 \mathrm{kDa}$. 


\section{Identification of Proteins Associated with ini-tRNA in the Nucleus Through Mass Spectrometry (MS) Analysis}

Using the successful incubation and affinity purification approach described above, we performed a largescale experiment, where we incubated nuclear extracts with non-radioactive biotinylated ini-tRNA (experimental samples) and non-biotinylated ini-tRNA (control). We also ran a parallel experiment under the same conditions but with biotinylated ${ }^{32} \mathrm{P}$-labeled ini-tRNA to help identify the relevant bands on the gel. We ran mass spectrometry (MS) analysis on the affinity-purified product (on bead digestion). Figure 2C summarizes the top results from three such biological repeats. To add stringency to our analysis, we considered genes as positive hits only when the ratio of biotin/no-biotin was larger than 3 , with p-value $<0.05$, and when at least two peptides were identified (for the complete list see Table S1). According to the MS analysis, the top candidate protein was nucleolin (NCL). Our MS run identified eighteen of its peptides ( $p$-value: 0.00086) and also peptides from the LOC397919 protein, another variant of NCL. Two additional MS experiments confirmed this result. In one of these two additional experiments, we extracted the proteins from the $60 \mathrm{kDa}$ and $120 \mathrm{kDa}$ bands and identified NCL in the $120 \mathrm{kDa}$ band. It should be noted that NCL, with its acidic N-terminus, four RNA binding motifs in the middle, and a glycine/arginine-rich C-terminus, undergoes multiple post-translational modifications such as acetylation, ADP-ribosylation, glycosylation, methylation, and phosphorylation $(34,35)$. These modifications affect its migration on SDS gels, giving a band of apparent $\mathrm{MW}$ of $120 \mathrm{kDa}$ (36). This altered migration is consistent with the migration of the upper band in our experiments (Figures 1, 2).

\section{Confirmation of NCL as a Candidate Protein Through Western Blot}

To further validate the association of NCL of apparent MW of $120 \mathrm{kDA}$ with ini-tRNA, we followed our UV crosslinking and affinity purification protocol (see Figures 2D,E) with western blot (WB) using antiNCL antibodies (Figure 2F). We identified NCL in the $120 \mathrm{kDa}$ band of untreated oocyte nuclei (germinal vesicle [GV] nuclei) (Figure 2F, lane 1), and in the $120 \mathrm{kDa}$ band of affinity-purified proteins crosslinked to ini-tRNA (Figure 2F, lane 3), but not in the control (Figure 2F, lane 2). These results embolden the support for the identification of NCL as a protein that directly and specifically associates with ini-tRNA only in the nucleus of Xenopus oocytes.

\section{Annotating the Genomic Landscape of Latent 5' Splice Sites}

To assess whether NCL is affecting regulation of splicing at latent 5' splice sites (here termed LSS), we first compiled a comprehensive annotation of these sites in the genome. We previously used a bioinformatics approach to identify potential LSSs in intronic sequences across the human genome, using data generated from expression exon microarrays (15). Using stringent criteria, we showed that LSSs are present in most human introns represented on the array (15). Here we used a more inclusive scoring threshold (MaxEntScan score (37) of 0 for intronic GT dinucleotides) to compile a comprehensive set of potential LSSs, filtered by known splice sites from multiple human gene annotation databases (see Materials and Methods for explanation, Figures 3A,B). This score threshold was chosen because it excluded the majority of intronic GT dinucleotides (less than 20\% of intronic GTs score higher than 0) but was inclusive of potential new LSSs because more than $98.5 \%$ of annotated 5'SSs score higher than 0 (Figure S1). Limiting our search to LSSs located $<1 \mathrm{~kb}$ downstream of annotated 5'SSs, we found a total of 1,381,214 LSSs (see Materials and Methods; Table S2). The majority of protein coding genes (nearly 89\%) contained at least one LSS, with an average of nearly 80 LSSs per gene (median value: 55). The genes with the highest number of LSSs were NBPF14 and NOTCH2NLB, which contained 1,190 LSSs each, whereas 72 other genes contained only one LSS. In general, we observed between 1 and 93 LSSs (median count: 8) within $1 \mathrm{~kb}$ downstream of annotated 5'SSs, with multiple LSS occurrences being typical (in nearly $95 \%$ of cases).

Most LSSs had lower MaxEntScan scores than the annotated 5'SSs upstream of them (median values of 3.19 and 8.68, respectively), and their score distribution was strongly skewed toward lower values (Figure 3C). However, if only the highest scoring LSSs downstream of each 5'SS was considered, the MaxEntScan score distribution was similar to the distribution of scores of annotated 5'SSs, albeit slightly shifted toward lower values (Figure 3C), highlighting the functional potential of intronic GT dinucleotides. For more than one third of established 5'SSs, there was at least one LSS downstream that scored higher (Figure 3D), highlighting the importance of the SOS mechanism for suppressing competition from these LSSs. The use of the highest scoring LSS instead of the annotated 5'SSs extended the upstream exon by an average of $469 \mathrm{bp}$ (Figure 3E) and incorporated an in frame STOP codon. In all but $15.5 \%$ of cases with multiple LSS occurrences, the highest scoring LSS was preceded by LSSs with weaker MaxEntScan scores. 


\section{NCL Knockdown by siRNA}

To further explore the role of NCL in SOS, we knocked down NCL in HEK-293 cells using siRNA in two biological replicate samples (referred to as NCLsi samples), from which we isolated proteins and RNA (see Materials and Methods). Our controls were from two paired biological replicate samples treated with nontargeting siRNA (referred to as CONTsi), and untreated cells (referred to as CONT). Western blot analysis indicated that the NCL protein level was knocked down 25-fold (Figure 4A). HiSeq RNA sequencing (RNA-Seq) analyses yielded more than 230 million reads (126-bp, paired-end) for each sample, and about $84 \%$ of them were uniquely aligned to the hg19 assembly (Table S3). Standard differential expression analysis by DESeq 2 revealed that NCL was the gene with the highest decrease in expression and the greatest fold change, as expected (Table S4). The normalized read counts for the NCL locus were reduced by more than 15-fold upon NCL knockdown (see Figure 4B; the DESeq2 model-based estimate of NCL downregulation is nearly 10 -fold, FDR adjusted $\mathrm{p}=3.5 \times 10^{-44}$ ). Therefore, the WB and RNA-Seq analyses consistently indicated successful knockdown of NCL.

\section{NCL Knockdown Activates Latent Splicing}

A detailed evaluation of the effect of NCL knockdown on splicing at LSSs was enabled by the depth of RNA sequencing. We used split reads (i.e., reads that align over two consecutive exons through an introninduced gap) as the basic measure for LSS usage. These reads connect LSSs with the downstream acceptor SSs and represent direct evidence for the splicing of the isoform of interest. We found more than 35,000 LSSs in each sample being supported by at least one split read (1-NCLsi: 43679; 2-CONTsi: 35865; 7NCLsi: 44124; 8-CONTsi: 41576). Overall, nearly 5\% of all LSSs are supported by at least one split read in at least one of the NCLsi samples (Figure 4C). After adjusting for differences in the total number of mapped reads for each sample, we found in each replicate an excess of $>4 \%$ (i.e. more than 1700) in the number of LSSs supported by split reads in NCLsi compared with CONTsi, supporting a role for NCL in regulating splicing at LSSs. To verify that NCL knockdown is linked to activation of splicing at many LSSs, we established a computational pipeline (Figure 4C) to separate cases of bona fide LSS activation from cases that are technical (e.g., spurious alignments) or biological (e.g., unannotated exons) artefacts. To define activated LSS, we required the NCLsi samples to exhibit increased usage of the LSS relative to the annotated upstream 5'SS, as well as higher levels of mapped reads throughout the exon extension region when compared to CONTsi samples (see Materials and Methods). We identified 399 LSSs in 362 genes that conform to all selection criteria (Table S5). The MaxEntScan score distribution for these 399 activated LSSs mimicked the score distribution for the 385 corresponding 5'SSs, with a slight shift toward smaller values (Figure 4D). Notably, 38.7\% of activated LSSs showed higher or equal MaxEntScan scores to the corresponding upstream 5'SS (Figures 4E, 5B). This finding, together with the similarity of the score distribution of activated LSSs to that of annotated 5'SSs, indicates that activated LSSs resemble genuine, annotated 5'SSs. Activated LSSs lead to exon extensions that range between 4 and $983 \mathrm{nt}$, with a median value of $81 \mathrm{nt}$ (Figure 4F). Three cases of activated LSSs are illustrated in Figures 4G-I, and a median profile of the increased expression throughout the exon extension region for all 399 cases of activated LSSs is shown in Figure 4J.

The heatmaps (Figure 5) provide a comprehensive view of the increase in expression associated with all activated LSSs upon NCL knock down, illustrating the wide range of length distribution of activated latent exons (Figure 5A). Figure 5B displays the average fold difference of activation of latent exons upon NCL knock down, each standardized to a length of $100 \mathrm{nt}$. It also portrays the MaxEntScan score difference between the activated LSS and the corresponding upstream 5'SS. Four activated LSSs are located in the $N C L$ gene itself: three in intron 9 and one in intron 10. In Figure 5B, these four LSSs appear at the top of the graph, as they show the highest increase in expression levels of the exon extension regions.

We noted that LSS activation can be detected in isoforms with a wide range of expression levels. In a small number of cases, we observed increased usage (i.e., activation) of the same LSS within the context of different transcripts (i.e., the LSS is spliced with two different downstream 3'SSs, or with two different upstream 5'SSs). For example, an LSS located in intron 4 of HMGN1 (chr21:40719840) appeared activated in two isoforms whose expression levels differ by almost 30 -fold between the isoforms.

\section{Gene Transcripts Affected by NCL Knockdown}

The cases with the strongest split read support for LSS activation after NCL knockdown are illustrated in Figure 6A (see also Table S5). This list includes proteins involved in several important cellular pathways and cell metabolism functions, such as transcription factors, oncogenes, kinases, splicing factors, translation 
factors, genes affecting cell motility, proliferation, and cellular trafficking, as well as NCL. These important functions can be found associated with many of the 362 genes affected by LSS activation, in agreement with the SOS being a general quality control mechanism.

For validation of the RNA-Seq results through an independent method, we selected nine gene transcripts (ANK2, TCERG1, LARS, WASHC5, COPA, RAF1, VRK2, HEATR1, and HTT) that are among the cases with the strongest RNA-Seq evidence for LSS activation (See Figure 6A). Using RT-PCR, we validated the RNA-Seq results showing that latent splicing was significantly elevated in response to knockdown of NCL as compared with the control cells (Figure 6B).

To learn whether LSS activation affects specific gene categories preferentially, we evaluated the over-representation of genes with activated LSSs among the gene sets included in all eight gene collections that make up the MSigDB database (i.e., numerous gene sets are within each collection). We found 27 gene sets with significant enrichment (after Bonferroni correction at gene collection level), 11 of which remained significant after stringent Bonferroni adjustment for the total number of 17,810 gene sets in MSigDB (Figure 6C, Table S6). The most significant $\left(\mathrm{p}=5.9 \times 10^{-10}\right)$ and largest overlap ( 84 genes) observed was for genes that encode RNA binding proteins according to their gene ontology annotation. Subsets of these genes make up large fractions of many of the other 27 gene sets with significant enrichments (Table S6), which could explain the diversity of gene sets significantly affected by LSS activation. Moreover, given that many RNA binding proteins have essential cellular roles that include repair of DNA damage, DNA replication, chromatin regulation, transcription, splicing, translation, protein folding, and cell proliferation, these results suggest that the SOS mechanism plays a critical role in protecting the integrity of cellular functions.

\section{Further Evidence Supporting the Relevance of NCL in SOS}

Our transcriptome survey through RNA-seq revealed increased latent splicing in hundreds of coding transcripts upon NCL knockdown. However, activation of latent splicing can be confounded and exacerbated by experimental stress $(15,26)$, such as the transfection procedure itself. To address this possibility, we switched sample labels between cases and controls and re-applied the computational pipeline to identify LSSs with increased usage. Under these conditions, we identified only 82 instances of LSSs activated in CONTsi compared to NCLsi samples (Tables 1, S7), nearly 5-fold fewer than identified with original sample labels. This finding indicates that NCL knockdown, rather than experimental stress, represents the main driver for the increase in latent splicing observed in NCLsi compared to control samples.

To investigate whether the role of NCL is specifically linked to the presence of PTCs, we analyzed cases of latent splicing where no PTCs are incorporated. Specifically, these cases have no in-frame STOP codon in the exon extension region, and the length of the exon extension is a multiple a three, so as not to change the reading frame. We identified a total of 43,702 such potential alternative donor SSs, referred to as adSS $_{3 n}$ (Table S8). Using the methodology described in Figure 4C for LSSs, we identified 50 cases of activated adSS 3 in the NCLsi samples compared to controls (Tables 1, S9). Upon label switching, we also identified 50 instances (Table S10), suggesting that NCL knockdown has little impact on the choice of alternative donor SSs when no PTCs are introduced. Indeed, comparison of the LSS and adSS $3 \mathrm{n}$ activation reveals a significant bias toward LSS activation in NCLsi samples, i.e. upon NCL knockdown (Table 1). The same effect was observed even with more stringent criteria for activation of latent splicing (Table 1), supporting the hypothesis of NCL involvement in a mechanism that prevents generation of PTC-containing mRNAs.

To further investigate the relationship between NCL knockdown and the presence of PTCs, we also analyzed cases of latent splicing where PTCs are incorporated in downstream exons through a shift of the reading frame. For this purpose we searched for alternative donor SSs that do not introduce PTCs in the exon extension, but extend the exon by lengths that are not multiple of three, which would consequently cause a reading frame shift and insertion of a PTC downstream. We found a total of 98,349 such potential alternative donor SSs, referred to as adSS $\mathrm{fs}_{\mathrm{fs}}$ (Table S11). Analysis of RNA-seq data revealed 221 cases of activated adSS $\mathrm{fs}_{\mathrm{fs}}$ in NCLsi compared to control samples (Tables 1, S12), and 52 activated adSS $\mathrm{fs}_{\mathrm{fs}}$ sites were found upon label switching (Tables 1, S13). No significant difference can be observed between activation patterns of LSSs and $\operatorname{adSS}_{\mathrm{fs}}(\mathrm{p}=0.28$, initial criteria; $\mathrm{p}=0.17$, stringent criteria, one-sided Fisher's exact test), supporting a general role for NCL in suppressing PTC-inducing splicing events. In contrast, these data reveal a significant bias toward activation of $\operatorname{adSS}_{\mathrm{fs}}$ compared to $\mathrm{adSS}_{3 \mathrm{n}}$ in NCLsi samples (i.e. upon NCL knockdown), similar to LSSs (Table 1). Therefore, our multiple complementary analyses support a general role of NCL in preventing PTC-harboring mRNAs. 


\section{Discussion}

\section{NCL is Directly Associated with ini-tRNA in the Nucleus}

Previous studies showed that ini-tRNA, which is associated with the endogenous spliceosome, play a role in SOS (20). Here we identified NCL as directly and specifically associated with ini-tRNA in the nucleus, and not in the cytoplasm. Through our UV crosslinking experiments, we identified factors that interact directly and specifically with ini-tRNA in the nucleus. The specificity of interaction of ini-tRNA with the bound factors was demonstrated by chase experiments using a hundred-fold excess of cold ini-tRNA. However, elongator-tRNA, which does not play a role in SOS, and is not associated with the endogenous spliceosome (20), when used at the same concentration (100-fold excess of cold elongator-tRNA) did not affect the interaction of ini-tRNA with the bound factors (Figure 1C, lane 3 and 4, respectively). Affinity purification and mass spectrometry analyses of the crosslinked components revealed NCL as a protein directly and specifically associated with ini-tRNA in the nucleus but not in the cytoplasm. The association is specific as demonstrated by the competition experiments. Furthermore, it should pointed out that although NCL is an abundant protein, the level of NCL protein expression in Xenopus ooctyes stage IV-V, used here, is low $(38,39)$. The identification of NCL as bound to ini-tRNA is in agreement with earlier findings of the association of NCL with the endogenous spliceosome (33). These findings indicate a role for NCL in splicing regulation, which we extend to further define a role in the SOS mechanism.

\section{NCL - a Multifunction Protein with Novel Role in the Spliceosome}

The results of our current study show that NCL has a previously undescribed role in splice site selection. NCL is known to perform multiple functions in numerous cell locations (nucleolus, cytoplasm, cell membrane and nucleoplasm) and to incur multiple post-translational modifications that affect its cellular location and function $(34,40)$. It is involved in the synthesis and maturation of ribosomes in the nucleolus, and while many of its functions in other cell components are described [e.g. a role in Pol II transcription, DNA repair, chromatin decondensation, and genome stability $(34,41,42)]$, most of the molecular details of its assorted functions are not understood. Furthermore, NCL is known to play a role in cancer, where its overexpression affects cell survival, proliferation, and invasion $(34,42,43)$. Our characterization of a previously unknown function for NCL is an important addition to the current body of knowledge of a protein crucial to cell function and involved in an important and widespread disease, cancer.

Recent studies have indicated a connection of NCL with splicing factors (44-47). We have previously found NCL as an integral component of the endogenous spliceosome (supraspliceosome), where it was identified associated with specific supraspliceosomes at all splicing stages (33). Furthermore, NCL was also identified to be associated with the general population of supraspliceosomes (48). While these published reports did not define a specific role for NCL in splicing regulation, as we do here, all of their findings support our discovery of NCL's role in splicing.

\section{NCL Proposed as a Novel Regulator of Splice Site Selection to Protect Cells from Insertion of PTC} Our RNA-seq analysis revealed activation of 399 LSSs when NCL was downregulated. It should be pointed out that the number of activated LSSs uncovered here is likely a conservative estimate, because all the latent mRNAs are PTC-bearing and would likely become substrates for downregulation by NMD, as was previously demonstrated (15), limiting their detection by RNA-seq. Notably, we were able to further validate a subset of 9/9 of these cases by RT-PCR (TCERG1, Transcription Elongation Regulator 1; ANK2, Ankyrin 2; LARS, Leucyl-tRNA Synthetase; COPA, Coatomer Protein Complex Subunit Alpha; WASHC5, WASH complex subunit 5; RAF1, Proto-Oncogene, Serine/Threonine Kinase; VRK2, vaccinia-related kinase (VRK) of serine/threonine kinase 2; HTT, Huntingtin; HEATR1, HEAT Repeat Containing 1). This validation provides support for our bioinformatic approach. Importantly, the MaxEntScan score distribution of activated LSSs closely resembles the distribution of genuine, annotated 5'SSs (Figure 4D). Furthermore, $38.7 \%$ of activated LSSs show higher or equal MaxEntScan scores to the corresponding upstream 5'SS (Figures 4E, 5B). For annotating potential LSSs we have used a rather relaxed MaxEntScan that covers 98.5\% of 5'SSs. Notably, even when more stringent MaxEntScan score thresholds are imposed for LSS selection a large fraction of activated LSSs can be detected. For example, a very stringent score threshold of 6.77, encompassing $80 \%$ of annotated 5'SSs, allows us to detect 191 activated LSSs (48\% of the activated LSSs). Even with this stringent criterion, we detect highly significant bias toward LSS activation compared to $\operatorname{adSS}_{3 n}\left(p=7.2 \times 10^{-7}\right.$, one-sided Fisher's exact test) upon NCL knockdown. These observations strongly 
support the involvement of NCL in a mechanism that selects splice sites and thereby prevents insertion of PTCs into mature transcripts.

The gene transcripts (see Figure 6A and Table S5) that underwent activation of latent splicing when NCL was downregulated encode for proteins that are involved in several important cellular pathways and cell metabolism functions; they include transcription factors, oncogenes, kinases, splicing factors, translation factors, and genes affecting cell motility, proliferation, and cellular trafficking, highlighting the importance of this regulation. This finding is consistent with previous studies both in magnitude and in functional diversity of affected genes (15). Interestingly, our gene set enrichment analysis revealed an enrichment of RNA binding proteins in many of the significant gene sets (Figure 6C, Table S6). On the one hand, this is surprising, because we show that LSSs are present in most genes, and NCL was identified as directly binding to ini-tRNA that is proposed to be a general component of the SOS mechanism that conveys the SOS components to the supraspliceosome (20). Also, a previous analysis of the effect of heat shock on SOS revealed activation of latent splicing in hundreds of gene transcripts, reflecting a wide repertoire of functional groups (15). On the other hand, this over-representation could be a reflection of the breadth of essential cellular functions performed by RNA binding proteins, and therefore, it is functionally critical that such genes are spliced properly. This is in line with recent observations that SS selection is tightly regulated in germ cells (49). Additional explanations for the observed enrichment in RNA binding proteins could include interaction with tissue-specific splicing factors or recruitment of splicing factors having high affinity for specific motifs within the pre-mRNA of RNA binding proteins. Yet, other explanations cannot be excluded at this stage. Further experiments directed at deciphering the molecular interactions are required to resolve this issue.

\section{Proposed Update of the SOS Speculative Model}

The proposed SOS working model (20) includes the role of ini-tRNA as an SOS factor. It invokes however a highly speculative sense triplet-recognition mechanism that can be interrupted by stop codon-binding proteins. In this study, we identified NCL as a potential novel SOS factor by demonstrating that NCL is directly associated with ini-tRNA in the nucleus and its downregulation activates latent splicing at hundreds of gene transcripts. Using these results, we have updated our working model to include SOS as a quality control mechanism within the supraspliceosome that acts before splicing (Figure 7). The supraspliceosome is composed of four native spliceosomes, which are connected by the pre-mRNA $(27,50,51)$. According to this model, first, splice site combinations are selected through the combinatorial interplay of positive and negative regulatory signals present in the pre-mRNA, which are recognized by trans-acting factors, resulting in the assembly of a supraspliceosome on each pre-mRNA (Figure 7A). Second, SOS endorses the right combinations of splice junctions (Figure 7B). The details of NCL role in SOS require further study.

We proposed that the SOS mechanism is based on three elements. First, the AUG sequence is recognized by the complementary anticodon (UAC) of the ini-tRNA, which is in a complex with auxiliary proteins (20). NCL can now be added in the model as a protein directly binding ini-tRNA, likely along with additional proteins. This step helps to establish a register for the recognition of the reading frame. The second SOS element involves the cooperative polymerization of protein(s) that bind triplets of nucleotides and in the absence of a PTC it reaches the selected 5'SS; this step is the quality control "confirming" step of SS selection that triggers the remodeling of the spliceosome to its functional state (Figure 7B, left). The final SOS element is suppression of splicing in the presence of a PTC, perhaps through a competing interaction with a stop-codon-binding protein (e.g., a release factor-like protein). The unproductive complex may undergo a conformational change and revert to the productive splicing complex involving the authentic 5'SS, as indicated by the double arrows (Figure 7B, right). In cases where the quality control mechanism fails, for example in cases of stress, downstream mechanism/s (e.g., NMD in the cytoplasm) may engage to safeguard the robust control of the system.

\section{Conclusions}

Our study identified NCL, a highly abundant and conserved protein, with multiple cellular functions involved in cancer, as a potential novel regulator of splice site selection and a component of the SOS mechanism that is proposed to protect cells from latent splicing that would generate transcripts with PTCs. We also identified novel splicing targets of NCL involved in multiple important cellular pathways and cell metabolism functions. 


\section{Materials and Methods}

Model system. We chose the Xenopus laevis (Xenopus) oocyte system for our experiments because SOS appears to be evolutionarily conserved (22), and thus the high protein concentration in Xenopus oocyte nuclei could be used to identify the proteins interacting with ini-tRNA in the nucleus. Furthermore, this system has been successfully used as a model system to study numerous biological processes, including splicing $(52,53)$, and we previously used this system to show that injected ini-tRNA, which functions in SOS, is uncharged with an amino acid (20).

In vitro Transcription of ini-tRNA. We generated a DNA template using pTRM, a plasmid carrying the WT ini-tRNA gene (54) and specific primers in a PCR reaction according to the manufacturer's instructions, and as detailed in Supplementary Methods. The RNA was purified using the miRNeasy kit (217004 Qiagen), according to the manufacturer's instructions, and analyzed on a denaturing gel.

In Vitro Transcription of Elongator-tRNA. We annealed $1 \mathrm{mM}$ of a primer having the elongator initRNA antiparallel sequence to $1 \mathrm{mM} \mathrm{T} 7$ primer $\left(90^{\circ} \mathrm{C}\right.$ for 3 minutes), and cooled it to room temperature (RT; annealing buffer: $10 \mathrm{mM}$ Tris- $\mathrm{HCl} \mathrm{pH} \mathrm{7.5,50} \mathrm{mM} \mathrm{NaCl}$ ). Then, we followed the transcription procedure explained above.

Injection of ini-tRNA into the Nuclei of Xenopus Oocytes. We extracted Xenopus oocytes as previously described $(55,56)$, and as detailed in Supplementary Methods. A day after oocyte extraction, $10 \mathrm{fmol}(20$ nl) of ${ }^{32} \mathrm{P}$ ini-tRNA was injected into the oocyte's nucleus (Picospritzer, PLI-100; Medical Systems Corp.). After 30 minutes, nuclei were extracted manually, and nuclei were gently squeezed out into GV buffer (5 mM HEPES, $17 \mathrm{mM} \mathrm{NaCl}, 83 \mathrm{mM} \mathrm{KCl}$ ). Each nucleus was gently washed and transferred to a new plate (57). Radioactivity of nuclei was measured (Perkin-Elmer Tri-Carbs 2900 TR Liquid Scintillation), and only nuclei having over half of the injected ini-tRNA were further assayed. Nuclei were irradiated at $4^{\circ} \mathrm{C}$ with an energy of 0.8 Joule (or 0.5 Joule as indicated) [Ultra-Lum UV Cross-Linker (UVC 515)]. Nuclei were digested by RNase A [1.75X10 $0^{-3}$ RNase A units (Sigma R-6513) per $50 \mu 1$ sample volume] and run on $8.7 \%$ SDS PAGE (1 nucleus/well).

In vitro Binding of ini-tRNA to Nuclear Extract of Xenopus Oocytes. We incubated nuclear extract (one nucleus per reaction, as described above) with increasing quantities $(0.5-2$ picomol) of biotinylated initRNA for 10 minutes and irradiated them with UV light (as described above).

Affinity Purification of Biotinylated ini-tRNA and its Crosslinked Factors. We affinity purified initRNA with crosslinked components using $5 \mu \mathrm{l}$ (or else as indicated) $\mathrm{C} 1$ streptavidin magnetic beads (Invitrogen 65001) in B\&W buffer according to the manufacturer's protocol. The pellet was resuspended in SDS loading buffer, boiled at $90^{\circ} \mathrm{C}$ for 5 minutes, and run on an $8.7 \%$ SDS PAGE.

RT-PCR Analysis. RT-PCR was performed on RNA extracted from the NCLsi and CONTsi treated cells as described (15), and detailed in Supplementary Methods, using the detailed sets of primers (see Supplementary Methods). The identity of all PCR products was confirmed by sequencing. Each experiment was repeated at least 3 times.

Western blot (WB). We ran our samples on $8.7 \%$ SDS PAGE and transferred them to a nylon membrane as described before (33). We performed WB analyses using anti-NCL antibodies (C23, cat \# Sc-13057, Enco) and visualized our product with horseradish peroxidase conjugated to affinity-purified goat antirabbit IgG (1:5000 dilution; H + L; Jackson ImmunoResearch).

Mass Spectrometry Analyses. We incubated 60 Xenopus oocyte nuclei with 120 picomol ini-tRNA (assay samples were prepared with biotinylated UTP and control samples were prepared with non-biotinylated UTP). All samples were affinity purified using $150 \mu \mathrm{C} 1$ streptavidin magnetic beads (as described above) and then resuspended in $100 \mu 110 \mathrm{mM}$ Tris buffer $\mathrm{pH}$ 7.5. We used in-solution, on-bead, tryptic digestion to prepare samples, as detailed in Supplementary Methods. Liquid Chromatography and Mass Spectrometry analyses are detailed in Supplementary Methods. 
Knockdown of NCL. We transfected siRNA targeted to NCL (L-003854-00-0005, ON-TARGETplus Human NCL (4691) siRNA- SMARTpool, Dharmacon), and non-targeting siRNA as control (D-00181001-05 ON-TARGETplus Non-targeting siRNA \#1, Dharmacon) into HEK 293 cells with TransIT-X2 (mcMIR-6003, Mirus) according to the manufacturer's instructions with some modifications. Cells grown in 6well plates were transfected with $45 \mathrm{nM}$ siRNA by using TransIT-X2 transfection reagent. After $24 \mathrm{~h}$, medium was changed, and cells were transfected again, as before. After $48 \mathrm{~h}$, total proteins and RNA were extracted (RNeasy Qiagen 74104) according to the manufacturer's instructions and analyzed.

RNA Library Preparation and Sequencing. Using the TruSeq ${ }^{\circledR}$ Stranded mRNA Sample Preparation kit (Illumina), we prepared a RNA library from four samples: (1)1-NCL-siRNA (1-NCLsi), (2)2-si-CONTROL (2-CONTsi), (3)7-NCL-siRNA (7-NCLsi), and (4)8-si-CONTROL (8-CONTsi). Briefly, polyA fraction (mRNA) was purified from $500 \mathrm{ng}$ of total RNA for each sample, fragmented, and used to generate double stranded cDNA. We then performed end repair, A base addition, adapter ligation, and PCR amplification of the samples. We evaluated the libraries with Qubit and TapeStation. Sequencing libraries were constructed with barcodes to allow multiplexing of four samples on four sequencing lanes, which were then sequenced with the Illumina HiSeq 2500 V4 instrument. More than 234 million paired-end, 126-bp reads were generated in total for each sample (Table S3).

Identification of Latent 5'SSs and Alternative Donor SSs. We compiled a comprehensive list of potential latent 5' splice sites (LSSs) in the human genome by investigating all GT dinucleotides located in intronic regions of multi-exon coding transcripts. Upon passing a MaxEntScan score threshold of 0 , the main criterion qualifying a GT dinucleotide as an LSS is the introduction of at least one in-frame STOP codon upstream (see Supplementary Methods for detailed information). Other alternative donor splice sites, such as adSS 3 (extend coding exons by lengths that are multiple of three) and adSS $\mathrm{fs}_{\mathrm{fs}}$ (extend coding exons by lengths that are not multiple of three), were selected only among GT dinucleotides that do not introduce upstream in-frame STOP codons.

Analysis of RNA-Seq Data. We performed read alignment with the STAR (v2.3.1) aligner (58) against the hg19 (GRCh37) assembly of the human genome (for further details see Supplementary Methods). We used the QoRTs package (59) to quantify reads mapped to exon junctions and genes included in the Gencode v19 annotation track. Differential gene expression analysis was performed with the DESeq2 package (60) in R (v3.2.2) with two case samples (1-NCLsi and 7-NCLsi) and two controls (2-CONTsi, 8-CONTsi).

Quantification of Increased LSS Usage. To compare LSS usage between samples we used the counts of reads mapped to the junction formed by the LSS and the downstream acceptor SS normalized by the counts of reads mapped to the corresponding canonical junction (i.e. \# split-reads $_{\mathrm{LSS}} /$ \# split-reads $_{5}$ 'ss). As candidates for activation of latent splicing we considered those LSSs for which we observed at least one NCLsi replicate with at least 4 reads supporting the LSS and a fold increase $\geq 1.5$ in normalized LSS usage in the NCLsi compared to the CONTsi sample. Additional conditions imposed to minimize the number of false positives are described in the Supplementary Methods.

Analysis of Expression Profiles. To further verify that the observed increase in LSS usage corresponds to the extension of the upstream exon into the intronic region we built expression profiles by quantifying reads mapped throughout the exon extension region. We compared normalized NCLsi and CONTsi profiles and retained cases for which both replicates were consistent with the extension of the upstream exon using two main criteria: $i$ ) reads were present throughout the exon extension region in the NCLsi sample (but they can be absent from the CONTsi sample), and ii) normalized expression levels were consistently higher in NCLsi compared to CONTsi samples throughout the exon extension region. For further details, see Supplementary Methods.

Gene Set Analysis. Overall, LSSs were assigned to a total of 17,975 Entrez gene IDs, with only 7,287 LSSs $(0.5 \%)$ remaining unassigned. Enrichment of various biological properties among genes with activated LSSs was evaluated with the hypergeometric test against curated gene sets from the Molecular Signatures Database (Version 6.2; MSigDB) $(61,62)$. Additional details are available in the Supplementary Methods. 


\section{Acknowledgments}

We thank Dr. Y. Nevo for helpful discussions and suggestions, A. Petcho for excellent technical assistance, and Karoun Bagamian for editing services. This study was partially supported by grants from the Israel Science Foundation (ISF), The Israel National Center for Personalized Medicine (INCPM), and the Israel Cancer Research Fund (ICRF) to R.S., and the Helen and Milton Kimmelman Center for Biomolecular Structure and Assembly at the Weizmann Institute of Science (to J.S.). We thank The Mantoux Institute for Bioinformatics, The Crown Institute for Genomics, and The de Botton Institute for Protein Profiling of The Nancy and Stephen Grand INCPM, Weizmann Institute of Science, for mass spectrometry and analysis, and for RNA sequencing. V.G. and L.E. were supported by the Intramural Research Program of the National Human Genome Research Institute through funding granted to L.E. (1ZIAHG200323-14).

\section{Author Contributions}

J.S. and R.S. conceived and designed the study. K.S. and A.B. performed the experiments. Y.B.C. performed the injections to Xenopus oocytes. R.S., V.G., and L.E. designed the RNA-Seq experiments. V.G. and L.E. designed the bioinformatic analyses. V.G. performed the bioinformatic analyses, including analysis of the RNA-Seq data. R.S., V.G., and L.E. wrote the paper. All authors discussed findings and commented on the final manuscript.

\section{Competing Interest Statement}

The authors declare no competing interests. 


\section{References}

1. O. Kelemen et al., Function of alternative splicing. Gene 514, 1-30 (2013).

2. Y. Lee, D. C. Rio, Mechanisms and regulation of alternative pre-mRNA splicing. Annu. Rev. Biochem. 84, 291-323 (2015).

3. M. Akerman et al., Differential connectivity of splicing activators and repressors to the human spliceosome. Genome Biol. 16, 119 (2015).

4. P. Papasaikas, J. Valcarcel, The spliceosome: the ultimate RNA chaperone and sculptor. Trends Biochem. Sci. 41, 33-45 (2016).

5. A. Fiszbein, A. R. Kornblihtt, Alternative splicing switches: important players in cell differentiation. Bioessays 39 (2017).

6. H. Dvinge, Regulation of alternative mRNA splicing: old players and new perspectives. FEBS Lett. 592, 2987-3006 (2018).

7. R. K. Singh, T. A. Cooper, Pre-mRNA splicing in disease and therapeutics. Trends Mol. Med. 18, 472-482 (2012).

8. M. Irimia, B. J. Blencowe, Alternative splicing: decoding an expansive regulatory layer. Curr. Opin. Cell Biol. 24, 323-332 (2012).

9. B. Chabot, L. Shkreta, Defective control of pre-messenger RNA splicing in human disease. J. Cell Biol. 212, 13-27 (2016).

10. E. El Marabti, I. Younis, The cancer spliceome: Reprograming of alternative splicing in cancer. Front. Mol. Biosci. 5, 80 (2018).

11. E. T. Wang et al., Alternative isoform regulation in human tissue transcriptomes. Nature 456, 470476 (2008).

12. N. Sheth et al., Comprehensive splice-site analysis using comparative genomics. Nucleic Acids Res. 34, 3955-3967 (2006).

13. S. A. Filichkin et al., Genome-wide mapping of alternative splicing in Arabidopsis thaliana. Genome Res. 20, 45-58 (2010).

14. E. Miriami, U. Motro, J. Sperling, R. Sperling, Conservation of an open-reading frame as an element affecting 5' splice site selection. J. Struct. Biol. 140, 116-122 (2002).

15. Y. Nevo et al., Genome-wide activation of latent donor splice sites in stress and disease. Nucleic Acids Res. 40, 10980-10994 (2012).

16. I. Behm-Ansmant et al., mRNA quality control: an ancient machinery recognizes and degrades mRNAs with nonsense codons. FEBS Lett. 581, 2845-2853 (2007).

17. B. Li et al., Stop codons affect 5' splice site selection by surveillance of splicing. Proc. Natl. Acad. Sci. USA 99, 5277-5282 (2002).

18. C. Wachtel, B. Li, J. Sperling, R. Sperling, Stop codon-mediated suppression of splicing is a novel nuclear scanning mechanism not affected by elements of protein synthesis and NMD. RNA 10, 1740-1750 (2004).

19. E. Kamhi et al., AUG sequences are required to sustain nonsense-codon-mediated suppression of splicing. Nucleic Acids Res. 34, 3421-3433 (2006).

20. E. Kamhi, O. Raitskin, R. Sperling, J. Sperling, A potential role for initiator-tRNA in pre-mRNA splicing regulation. Proc. Natl. Acad. Sci. USA 107, 11319-11324 (2010).

21. E. Miriami, J. Sperling, R. Sperling, Heat shock affects 5 ' splice site selection, cleavage and ligation of CAD pre-mRNA in hamster cells, but not its packaging in lnRNP particles. Nucleic Acids Res. 22, 3084-3091 (1994).

22. Y. Nevo, J. Sperling, R. Sperling, Heat shock activates splicing at latent alternative 5 ' splice sites in nematodes. Nucleus 6, 225-235 (2015).

23. E. D. Karousis, S. Nasif, O. Muhlemann, Nonsense-mediated mRNA decay: novel mechanistic insights and biological impact. Wiley Interdiscip. Rev. RNA 7, 661-682 (2016).

24. T. Kurosaki, L. E. Maquat, Nonsense-mediated mRNA decay in humans at a glance. J. Cell. Sci. 129, 461-467 (2016).

25. F. He, A. Jacobson, Nonsense-mediated mRNA decay: degradation of defective transcripts is only part of the story. Annu. Rev. Genet. 49, 339-366 (2015).

26. J. Sperling, R. Sperling, Nuclear surveillance of RNA polymerase II transcripts. RNA Biol. 5, 220224 (2008). 
27. R. Sperling, Small non-coding RNA within the endogenous spliceosome and alternative splicing regulation. Biochim. Biophys. Acta Gene. Regul. Mech. 1862, 194406 (2019).

28. O. Mühlemann et al., Precursor RNAs harboring nonsense codons accumulate near the site of transcription. Mol. Cell 8, 33-44 (2001).

29. S. Aoufouchi, J. Yelamos, C. Milstein, Nonsense mutations inhibit RNA splicing in a cell-free system: recognition of mutant codon is independent of protein synthesis. Cell 85, 415-422 (1996).

30. A. Gersappe, L. Burger, D. J. Pintel, A premature termination codon in either exon of minute virus of mice P4 promoter-generated pre-mRNA can inhibit nuclear splicing of the intervening intron in an open reading frame-dependent manner. J. Biol. Chem. 274, 22452-22458 (1999).

31. V. de Turris, P. Nicholson, R. Z. Orozco, R. H. Singer, O. Mühlemann, Cotranscriptional effect of a premature termination codon revealed by live-cell imaging. $R N A$ 17, 2094-2107 (2011).

32. K. Shefer, J. Sperling, R. Sperling, The supraspliceosome - a multi-task machine for regulated premRNA processing in the cell nucleus. Comput. Struct. Biotechnol. J. 11, 113-122 (2014).

33. H. Kotzer-Nevo, F. de Lima Alves, J. Rappsilber, J. Sperling, R. Sperling, Supraspliceosomes at defined functional states portray the pre-assembled nature of the pre-mRNA processing machine in the cell nucleus. Int. J. Mol. Sci. 15, 11637-11664 (2014).

34. C. M. Berger, X. Gaume, P. Bouvet, The roles of nucleolin subcellular location in cancer. Biochimie 113, 78-85 (2015).

35. K. Abdelmohsen, M. Gorospe, RNA-binding protein nucleolin in disease. RNA Biol. 9, 799-808 (2012).

36. H. Ginisty, H. Sicard, B. Roger, P. Bouvet, Structure and functions of nucleolin. J. Cell. Sci. 112 (Pt 6), 761-772 (1999).

37. G. Yeo, C. B. Burge, Maximum entropy modeling of short sequence motifs with applications to RNA splicing signals. J. Comput. Biol. 11, 377-394 (2004).

38. K. Bicknell et al., Nucleolin is regulated both at the level of transcription and translation. Biochem. Biophys. Res. Commun. 332, 817-822 (2005).

39. M. Caizergues-Ferrer et al., Nucleolin from Xenopus laevis: cDNA cloning and expression during development. Genes Dev. 3, 324-333 (1989).

40. K. Abdelmohsen, M. Gorospe, RNA-binding protein nucleolin in disease. RNA Biol. 9, 799-808 (2012).

41. A. C. Gregorio et al., Meeting the needs of breast cancer: a nucleolin's perspective. Crit. Rev. Oncol. Hematol. 125, 89-101 (2018).

42. I. Ugrinova, M. Petrova, M. Chalabi-Dchar, P. Bouvet, Multifaceted nucleolin protein and its molecular partners in oncogenesis. Adv. Protein Chem. Struct. Biol. 111, 133-164 (2018).

43. W. Jia, Z. Yao, J. Zhao, Q. Guan, L. Gao, New perspectives of physiological and pathological functions of nucleolin (NCL). Life Sci. 186, 1-10 (2017).

44. V. Marchand et al., Identification of protein partners of the human immunodeficiency virus 1 tat $/$ rev exon 3 leads to the discovery of a new HIV-1 splicing regulator, protein hnRNP K. RNA Biol. 8, 325-342 (2011).

45. Y. Soeno et al., Identification of novel ribonucleo-protein complexes from the brain-specific snoRNA MBII-52. RNA 16, 1293-1300 (2010).

46. S. Das et al., Characterization of nucleolin K88 acetylation defines a new pool of nucleolin colocalizing with pre-mRNA splicing factors. FEBS Lett. 587, 417-424 (2013).

47. I. Ugrinova, M. Chalabi-Dchar, K. Monier, P. Bouvet, Nucleolin interacts and co-localizes with components of pre-catalytic spliceosome complexes. Sci 1, 33 (2019).

48. Y. I. Chen et al., Proteomic analysis of in vivo-assembled pre-mRNA splicing complexes expands the catalog of participating factors. Nucleic Acids Res. 35, 3928-3944 (2007).

49. I. Ehrmann et al., An ancient germ cell-specific RNA-binding protein protects the germline from cryptic splice site poisoning. Elife 8 (2019).

50. R. Sperling, The nuts and bolts of the endogenous spliceosome. Wiley Interdiscip. Rev. RNA 8, 8:e1377 (2017).

51. J. Sperling, R. Sperling, Structural studies of the endogenous spliceosome - The supraspliceosome. Methods 125, 70-83 (2017).

52. K. H. Moon, X. Zhao, Y. T. Yu, Pre-mRNA splicing in the nuclei of Xenopus oocytes. Methods Mol. Biol. 322, 149-163 (2006). 
53. A. Mereau, C. Le Sommer, H. Lerivray, M. Lesimple, S. Hardy, Xenopus as a model to study alternative splicing in vivo. Biol. Cell 99, 55-65 (2007).

54. T. V. Pestova et al., Molecular mechanisms of translation initiation in eukaryotes. Proc. Natl. Acad. Sci. USA 98, 7029-7036 (2001).

55. Y. Ben-Chaim, O. Tour, N. Dascal, I. Parnas, H. Parnas, The M2 muscarinic G-protein-coupled receptor is voltage-sensitive. J. Biol. Chem. 278, 22482-22491 (2003).

56. L. Ohana, O. Barchad, I. Parnas, H. Parnas, The metabotropic glutamate G-protein-coupled receptors mGluR3 and mGluR1a are voltage-sensitive. J. Biol. Chem. 281, 24204-24215 (2006).

57. E. H. Birkenmeier, D. D. Brown, E. Jordan, A nuclear extract of Xenopus laevis oocytes that accurately transcribes 5S RNA genes. Cell 15, 1077-1086 (1978).

58. A. Dobin et al., STAR: ultrafast universal RNA-seq aligner. Bioinformatics 29, 15-21 (2013).

59. S. W. Hartley, J. C. Mullikin, QoRTs: a comprehensive toolset for quality control and data processing of RNA-Seq experiments. BMC Bioinformatics 16, 224 (2015).

60. M. I. Love, W. Huber, S. Anders, Moderated estimation of fold change and dispersion for RNAseq data with DESeq2. Genome Biol. 15, 550 (2014).

61. A. Subramanian et al., Gene set enrichment analysis: a knowledge-based approach for interpreting genome-wide expression profiles. Proc. Natl. Acad. Sci. USA 102, 15545-15550 (2005).

62. A. Liberzon et al., The Molecular Signatures Database (MSigDB) hallmark gene set collection. Cell Syst. 1, 417-425 (2015).

63. M. Azubel, S. G. Wolf, J. Sperling, R. Sperling, Three-dimensional structure of the native spliceosome by cryo-electron microscopy. Mol. Cell 15, 833-839 (2004).

64. M. Azubel, N. Habib, J. Sperling, R. Sperling, Native spliceosomes assemble with pre-mRNA to form supraspliceosomes. J. Mol. Biol. 356, 955-966 (2006).

65. S. Cohen-Krausz, R. Sperling, J. Sperling, Exploring the architecture of the intact supraspliceosome using electron microscopy. J. Mol. Biol. 368, 319-327 (2007).

66. S. Müller et al., A supraspliceosome model for large nuclear ribonucleoprotein particles based on mass determinations by scanning transmission electron microscopy. J. Mol. Biol. 283, 383-394 (1998). 


\section{Figures}

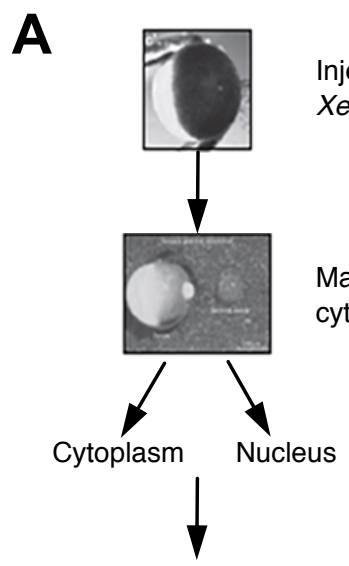

Inject P32-labeled ini-tRNA into Xenopus oocytes nucleus (GV)

Manually isolate nucleus and cytoplasm

UV crosslink nucleus and cytoplasm separately

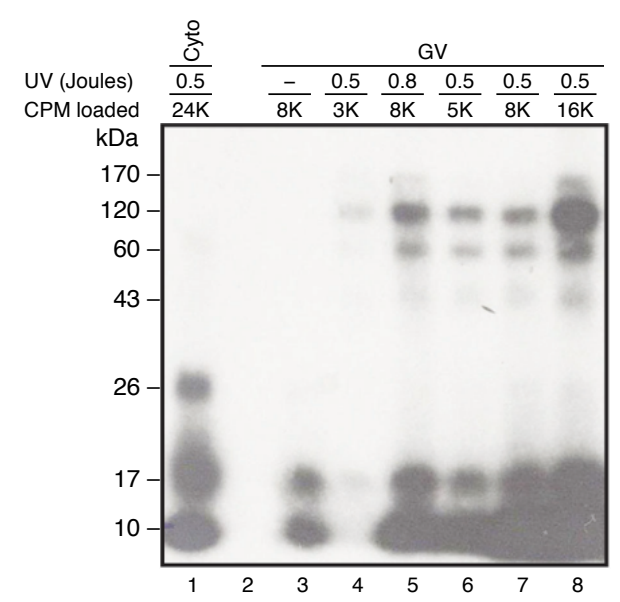

D

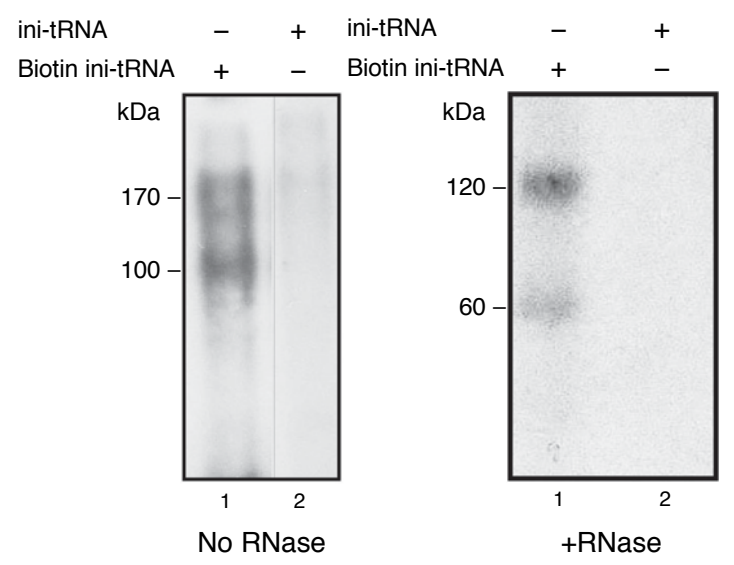

Figure 1. Specific crosslinking of proteins to ini-tRNA in the nucleus. $(\boldsymbol{A})$ Scheme of the experiment. In vitro transcribed ${ }^{32} \mathrm{P}$-labeled ini-tRNA was injected into individual nuclei of Xenopus oocytes. Isolated nuclei and cytoplasm were crosslinked with UV light, and the crosslinked complexes were further digested with RNase. (B) SDS PAGE analysis of aliquots from experiments performed as described in $A$. Lane 1, UV crosslinked cytoplasm; lane 3, non-crosslinked nucleus; lanes 4-8, individual nuclei transfected with the indicated levels of ini-tRNA (cpm) and crosslinked by UV light at the indicated dose. (C) Specific association with ini-tRNA. The UV crosslinked bands are chased by $100 \mathrm{X}$ cold ini-tRNA, but not by $100 \mathrm{X}$ cold elongator-tRNA. Lower panel, quantification of the experiment (4 biological repeats for panels 1-3, and 3 biological repeats for panel 4) (D) Affinity purification of proteins bound to ini-tRNA. In vitro transcribed biotinylated ${ }^{32} \mathrm{P}$-labeled ini-tRNA was injected into individual nuclei of Xenopus oocytes [as described in (A)], UV crosslinked, and affinity purified on streptavidin magnetic beads and run on SDS PAGE. Control, non-biotinylated ${ }^{32} \mathrm{P}-$ labeled ini-tRNA. 


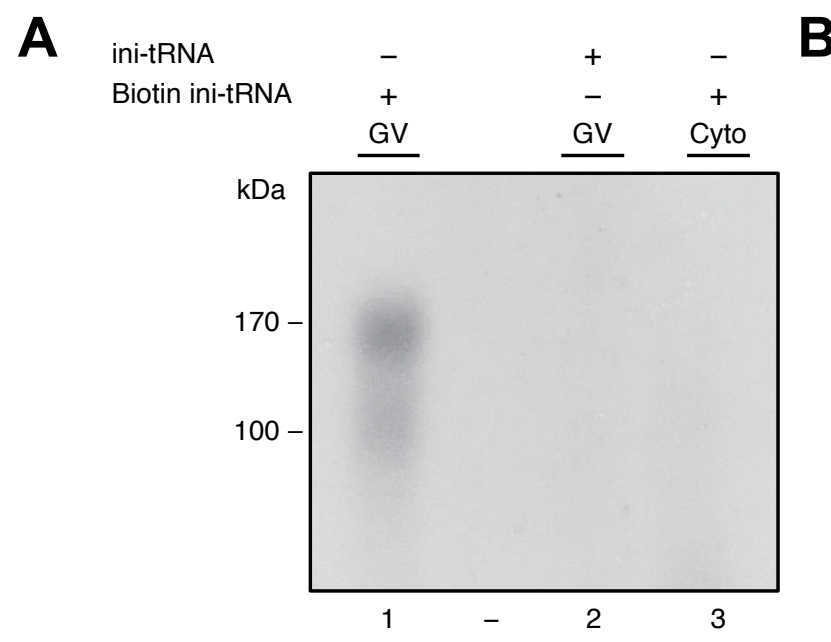

$$
\text { B }
$$

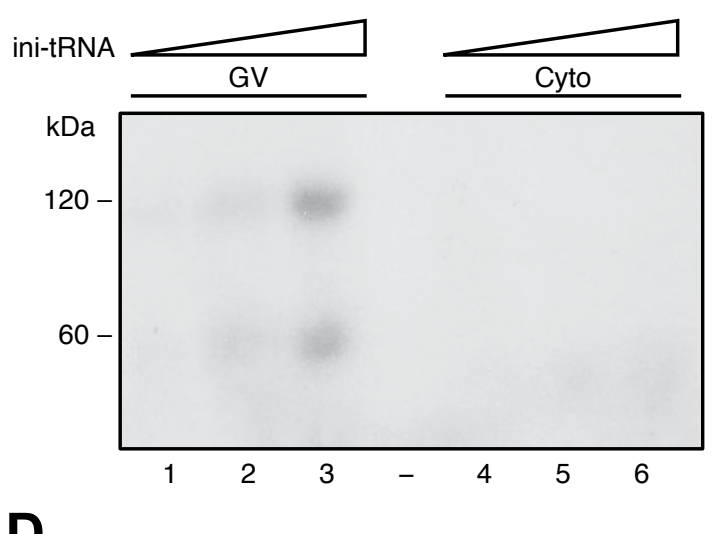

Incubation of Xenopus nuclear extract with biotinylated ini-tRNA

\begin{tabular}{|lccl|}
\hline $\begin{array}{l}\text { MS Top } \\
\text { Candidates }\end{array}$ & $\begin{array}{c}\text { Biotin/ } \\
\text { No Bio. }^{\text {a }}\end{array}$ & $\begin{array}{l}\text { No. } \\
\text { Pept. }^{b}\end{array}$ & $p$-value \\
NCL & 4.8 & 18 & 0.00086 \\
YWHAQ & 53.3 & 2 & 0.01268 \\
CIRBB & 2.9 & 1 & 0.01796 \\
$\begin{array}{l}\text { LOC307919 } \\
\text { (NCL) }\end{array}$ & 5.3 & 8 & 0.01910 \\
\hline
\end{tabular}

$\mathbf{E}$

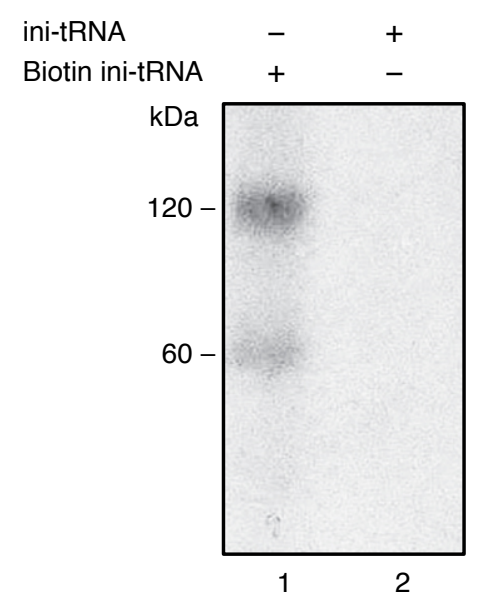

$\mathbf{F}$

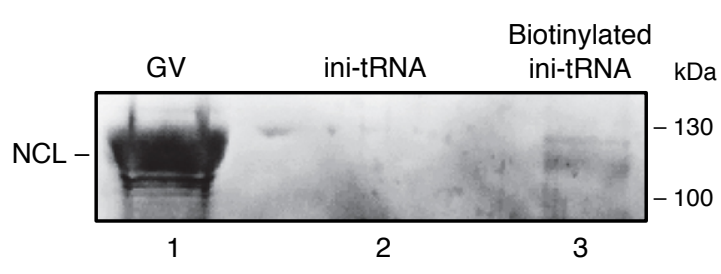

Figure 2. NCL is directly bound to ini-tRNA in the nucleus. $(\boldsymbol{A}, \boldsymbol{B})$ Proteins bound to ini-tRNA in nuclear extracts. Increasing quantities of in vitro transcribed biotinylated ${ }^{32} \mathrm{P}$-labeled ini-tRNA were incubated with nuclear (lanes 1-3) and cytoplasmic (lanes 4-6) extracts, UV crosslinked and affinity purified on streptavidin magnetic beads and run on SDS PAGE before $(A)$ or after $(B)$ RNase treatment. (C) Significant peptides associated with ini-tRNA in the nucleus based on mass spectrometry analysis; ${ }^{a} \mathrm{Bio} /$ noBio indicates the fold change in biotin versus no biotin, average of three biological experiments; ${ }^{b}$ number of peptides (see also Table S1). (D) Scheme of the experiment described in $E, F$ confirming NCL binding to ini-tRNA. (E) In vitro transcribed biotinylated ${ }^{32} \mathrm{P}$-labeled ini-tRNA was incubated with nuclear extract of Xenopus oocytes, UV crosslinked, and subjected to affinity purification on streptavidin magnetic beads, and run on SDS PAGE after RNase digestion (lane 1), and control non-biotinylated ${ }^{32} \mathrm{P}$-labeled ini-tRNA that went through the same procedure (lane 2). (F) Analogous experiment, followed by WB with anti-NCL antibodies. Left, GVs; middle, control non-biotinylated ini-tRNA; right, biotinylated ini-tRNA. 

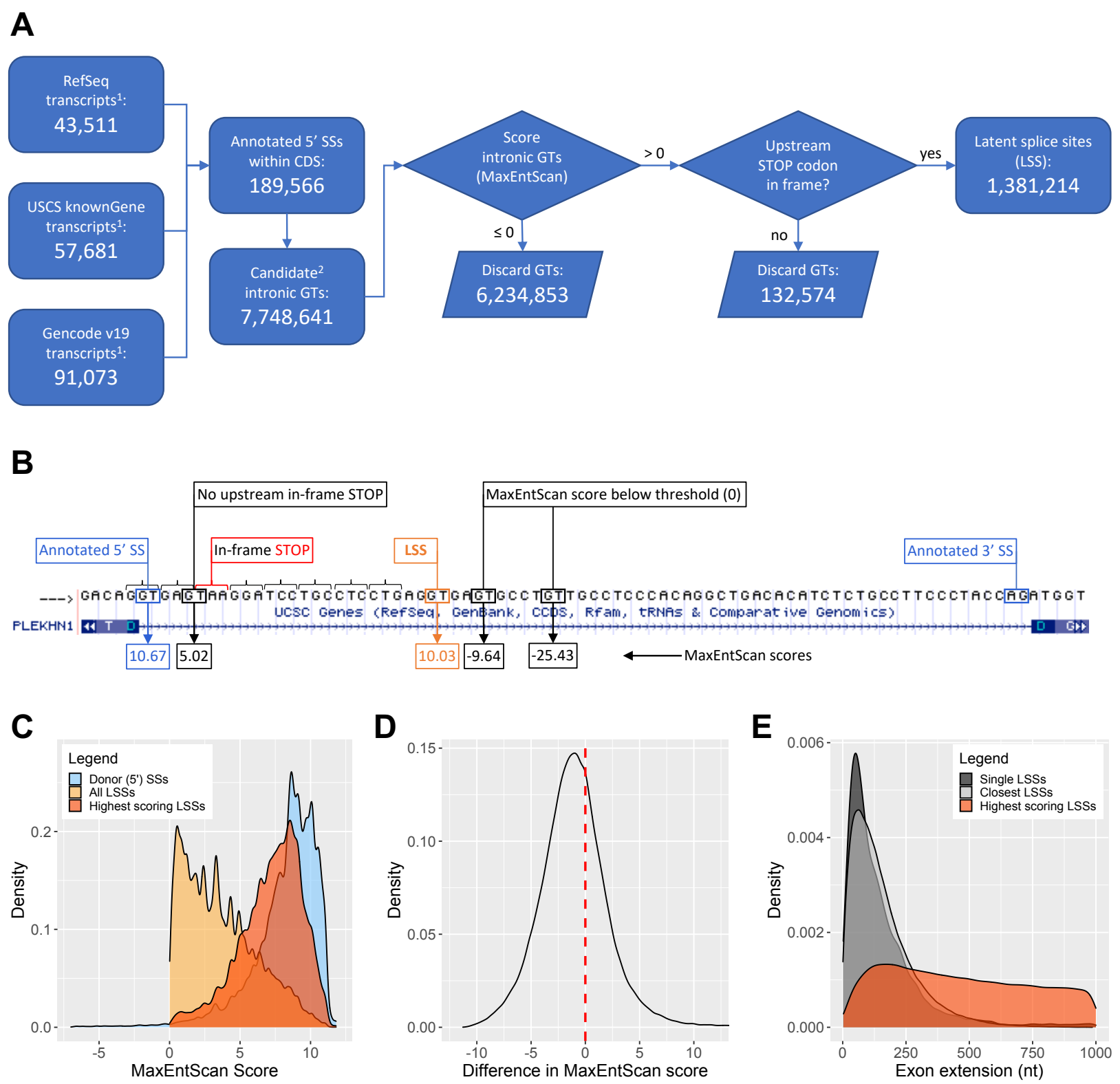

Figure 3. Latent splice sites (LSSs) in human genes. (A) Flowchart of computational steps performed to identify LSSs using the hg19 assembly of the human genome. ${ }^{1}$ Number of transcripts reflect only multipleexon protein-coding transcripts annotated on chromsomes $1-22, \mathrm{X}$, and Y. ${ }^{2}$ Candidate GTs include only those GTs located in whitin-CDS intronic regions that would extend the upstream exon by at most $1000 \mathrm{nt}$ while preserving a downstream intronic region of at least $20 \mathrm{nt}$ (see also Table S2). (B) Examples of GTs evaluated for their potential LSS function in intron 15 of the PLEKHN1 gene (RefSeq accession number NM 032129). (C) Distributions of MaxEntScan scores for LSSs and corresponding annotated donor 5'SSs. $0.9^{\circ}$ of annotated 5 'SSs have scores lower than -5 (not shown), and can be as low as -42.68 . (D) Distribution of differences in MaxEntScan scores between the highest scoring LSSs located downstream of an annotated 5'SS and the score of that 5'SS. 34.2\% of 5'SSs have at least one stronger LSS downstream of them. (E) Distributions of distances between LSSs and corresponding 5'SSs (i.e. exon extensions). "Single LSSs" denotes those cases where a single LSS can be found downstream of a specific 5'SS (8886 cases). For 5'SSs with more than one LSS downstream (159931 cases), distributions of exon extensions for both the closest and strongest LSSs are shown. 
A

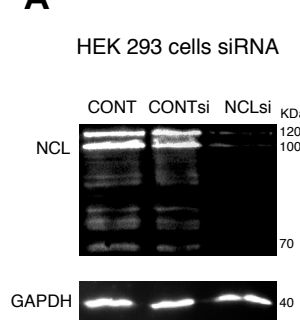

B

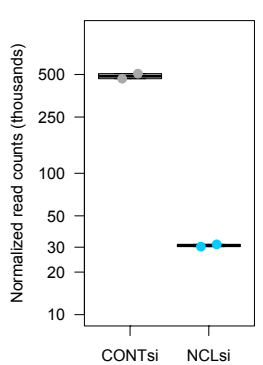

C

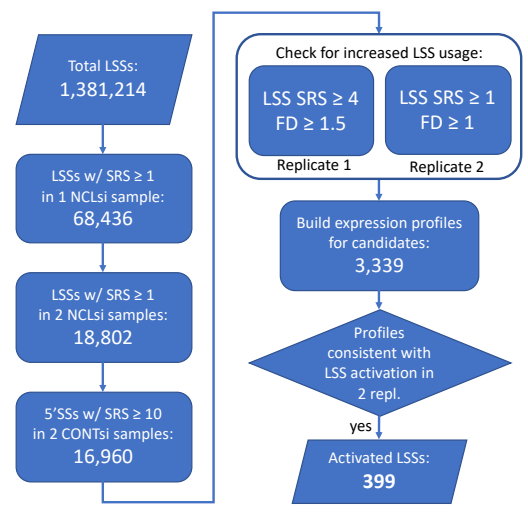

$\mathbf{F}$

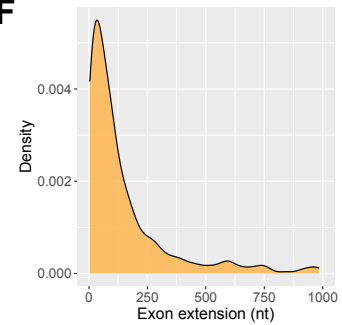

G

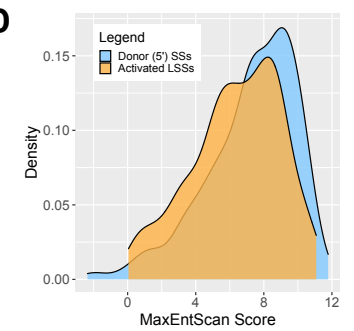

E

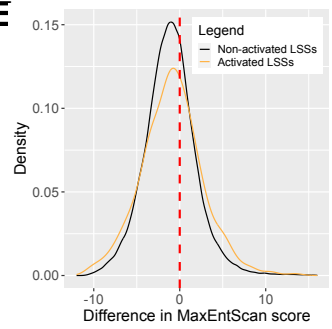

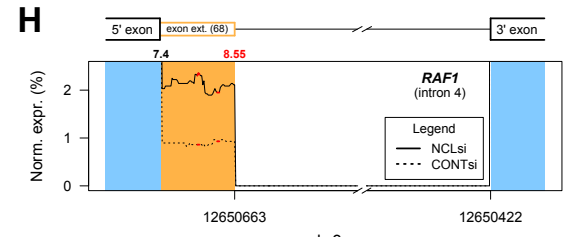

chr3

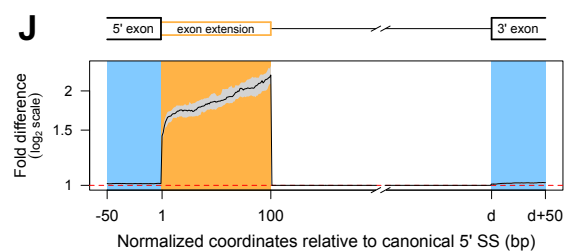

Figure 4. Identification of LSSs activated by knockdown of NCL. $(\boldsymbol{A}, \boldsymbol{B})$ HEK 293 cells were treated by siRNA against NCL (see Materials and Methods). As controls, we used non-targeting siRNA \#1(Dharmacon), and non-treated cells. Proteins and RNA were extracted after $48 \mathrm{hr}$. (A) Proteins were analysed by SDS PAGE. (B) Analysis of NCL expression using RNA-Seq data (see Materials and Methods). Normalized reads of RNA from cells treated by siNCL (NCLsi) and siControl (CONTsi) (biological duplicates from each) are presented (see also Table S4). (C) The computational pipeline for identification of activated LSSs. Selection of LSS activated candidates was done based on the presence of split reads supporting the LSS junction. Replicate 1 and 2 can refer to either sample replicate pairs. Expression profiles for all candidates were analyzed individually to assure consistency between the two replicates. SRS - split read support (see also Table S5). (D) Distribution of MaxEntScan scores for the 399 activated LSS and the corresponding 385 annotated 5'SS upstream of them. $(\boldsymbol{E})$ Distribution of differences in MaxEntScan scores between the activated LSSs and the corresponding upstream 5'SS (score ${ }^{\text {'sS }}$ - score $_{\text {LSS }}$ ). There are 401 unique LSS-5'SS pairs. Data for the set of non-activated LSSs was obtained with highest scoring LSSs downstream of 103,375 5'SSs with split read support of at least 10 reads for the canonical junction in both CONTsi samples. $(\boldsymbol{F})$ Distribution of lengths for exon extensions caused by LSS activation. (G-I) Typical expression profiles of exon extension regions upstream of LSSs activated in NCLsi samples. Positions of in-frame STOPs are shown by red segments. Numbers on top of the graph indicate MxEntScan scores for the annotated 5'SS (black) and the LSS (red). The length of the exon extension is indicated in the corresponding segment of the gene model. ( $\boldsymbol{J}$ ) Composite profile showing the fold difference in expression level between NCLsi and CONTsi samples for all 399 cases of activated LSSs. The black line represents median fold difference values at nucleotide resolution, whereas the gray area represents $95 \%$ confidence interval for the median. 
A

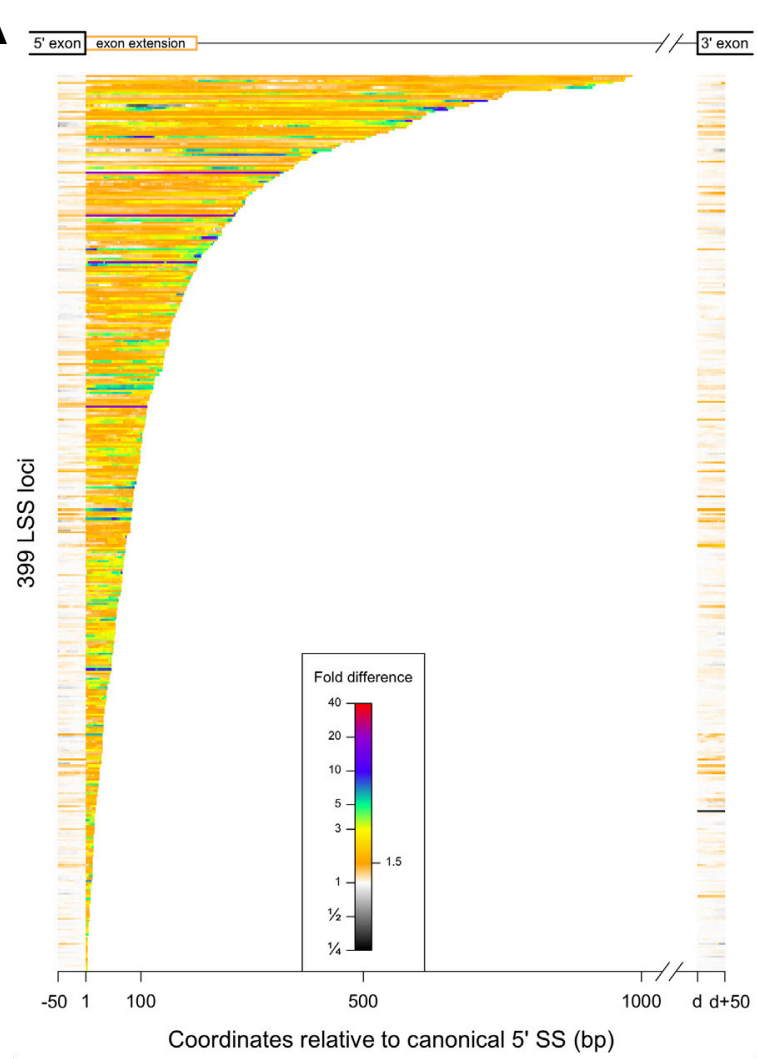

B

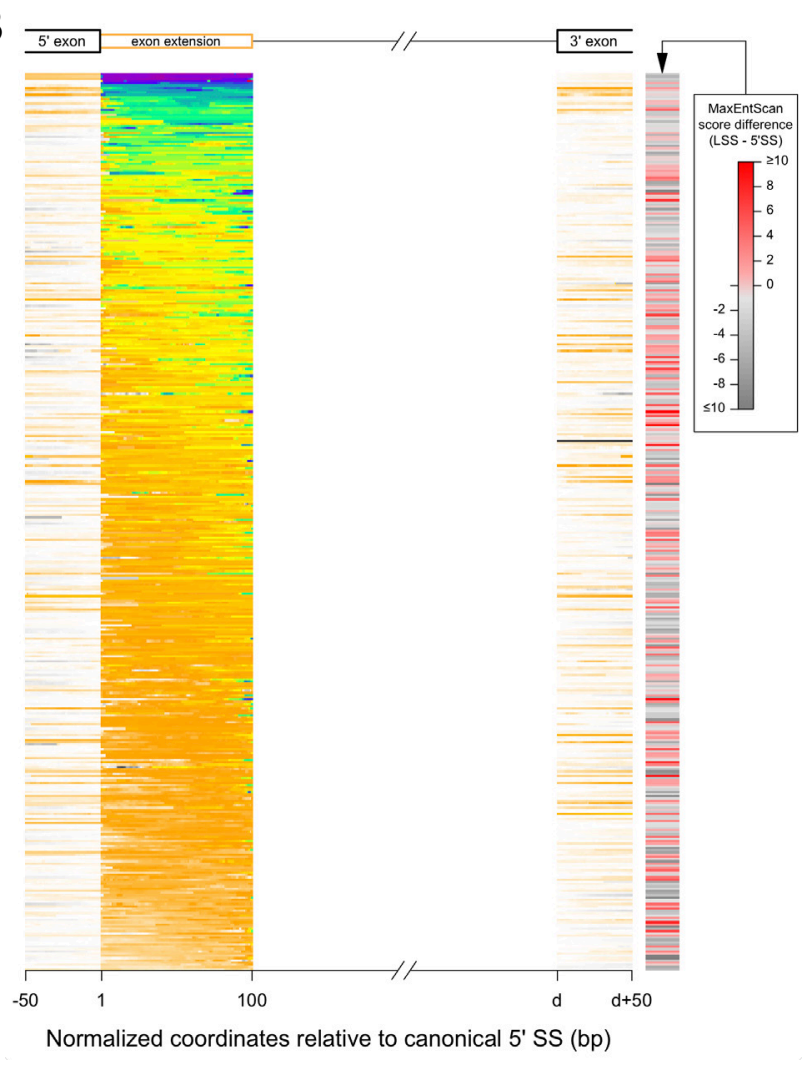

Figure 5. Overview of LSS activation upon NCL knockdown. Color-coded levels of fold-difference in expression levels is shown for all 399 activated LSSs. Fold difference was computed using normalized expression levels, where the normalization factor was the number of split reads supporting the corresponding annotated 5'SS (see also Table S5). (A) Data shown for actual length of exon extension regions, and LSSs are sorted based on the exon extension length. $(\boldsymbol{B})$ LSSs are sorted based on the average fold difference across the exon extension region, which is shown as a standardized length of $100 \mathrm{bp}$. The difference in MaxEntScan score between the LSS and the corresponding 5'SS is shown on the right. Shades of red correspond to cases where the LSS has a score higher or close to the score of the corresponding 5'SS. The score is not significntly correlated with the increase in expression observed for the exon extension (Spearman's $\rho=-0.051, \mathrm{p}=0.3$ ). 
A

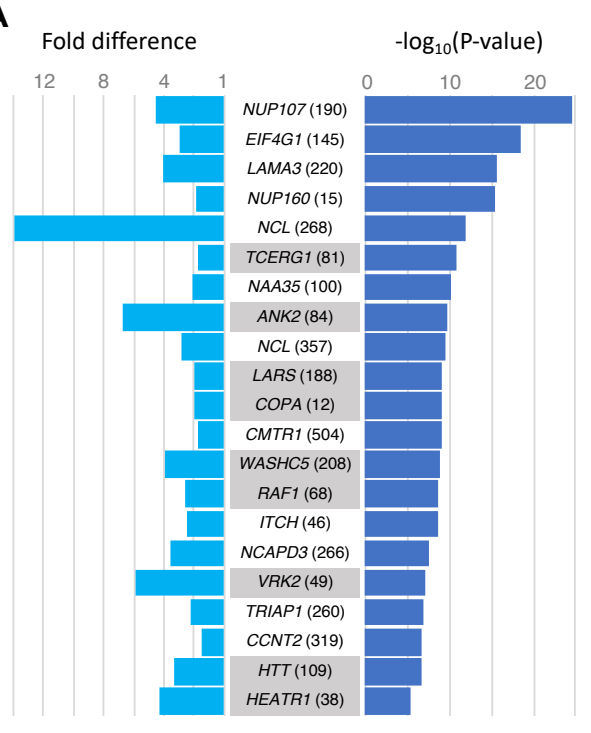

C

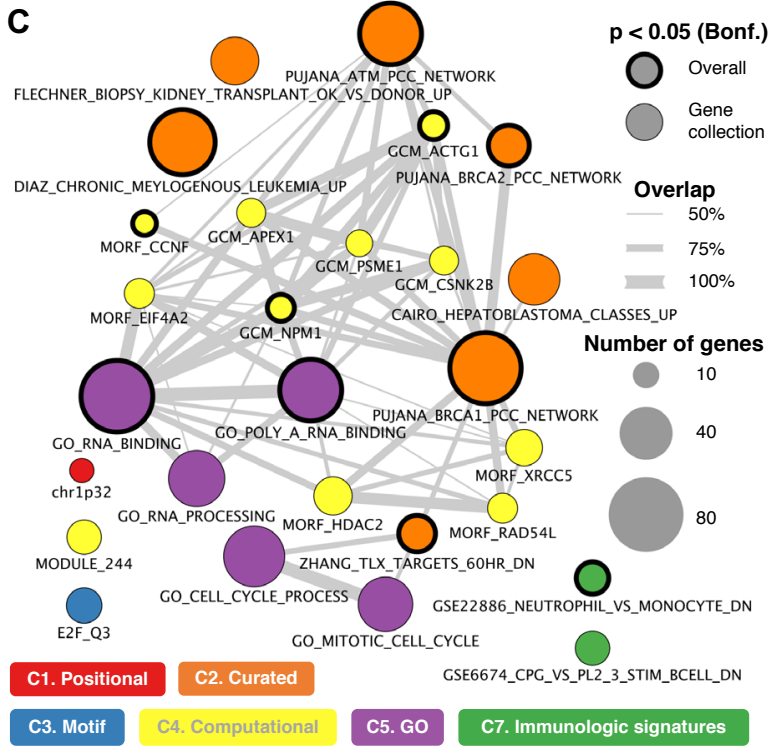

B

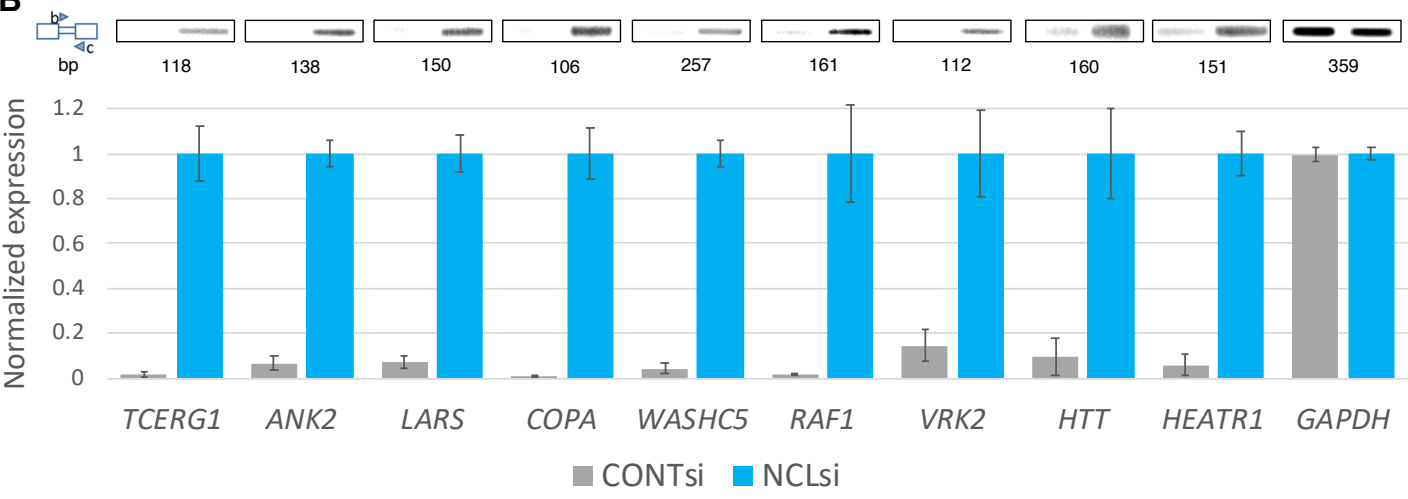

Figure 6. Genes with activated LSSs upon NCL knockdown. $(\boldsymbol{A})$ List of 20 activated LSSs with strongest support based on split reads from the RNA-Seq experiments. Cases are ranked based on the p-value obtained using one-sided Fisher's exact test with the number of reads supporting the LSS and the annotates 5'SS in the NCLsi and CTRLsi samples (values of the two replicates were combined using Fisher's method). The fold difference in normalized LSS usage between NCLsi and CTRLsi samples is shown in light blue (the value shown corresponds to the geometric mean between the two replicates). Name of the gene and length of the exon extension are also provided (complete details for these cases can be found in Table S5). Cases experimentally validated through RT-PCR are highlighted. The case in the HEATRl gene was ranked 32nd, but is included here to show it was also experimatally validated. (B) RT-PCR validation of activation of latent splicing at LSSs in nine genes expressed in NCLsi treated HEK 293T cells. CONTsi treated cell were used as control. Numbers below PCR bands represent the sizes of the PCR products obtained with primers designed to match the schematic representation shown on the left (boxes represent exons, narrow box represents latent exon, i.e. exon extension, triangles represent primers). Bars represent averages and SEMs for three biological replicates. TCERG1, Transcription Elongation Regulator 1; ANK2, Ankyrin 2; LARS, Leucyl-tRNA Synthetase; COPA, Coatomer Protein Complex Subunit Alpha; WASHC5, WASH complex subunit 5; RAF1, Proto-Oncogene, Serine/Threonine Kinase; VRK2, vaccinia-related kinase (VRK) of serine/threonine kinase 2; HTT, Huntingtin; HEATR1, HEAT Repeat Containing 1. GAPDH, glyceraldehyde-3-phosphate dehydrogenase was used for normalization. All PCR products were verified by sequencing. (C) Gene sets from MSigDB over-represented among the 362 genes with activated LSSs. Circle areas correspond to the number of genes. Gray lines connect gene sets that share at least $50 \%$ of the genes in the smaller set. Thick circle borders correspond to gene sets that remain significant after stringent overall Bonferroni correction for multiple testing (17810 total tests) (see also Table S6). 

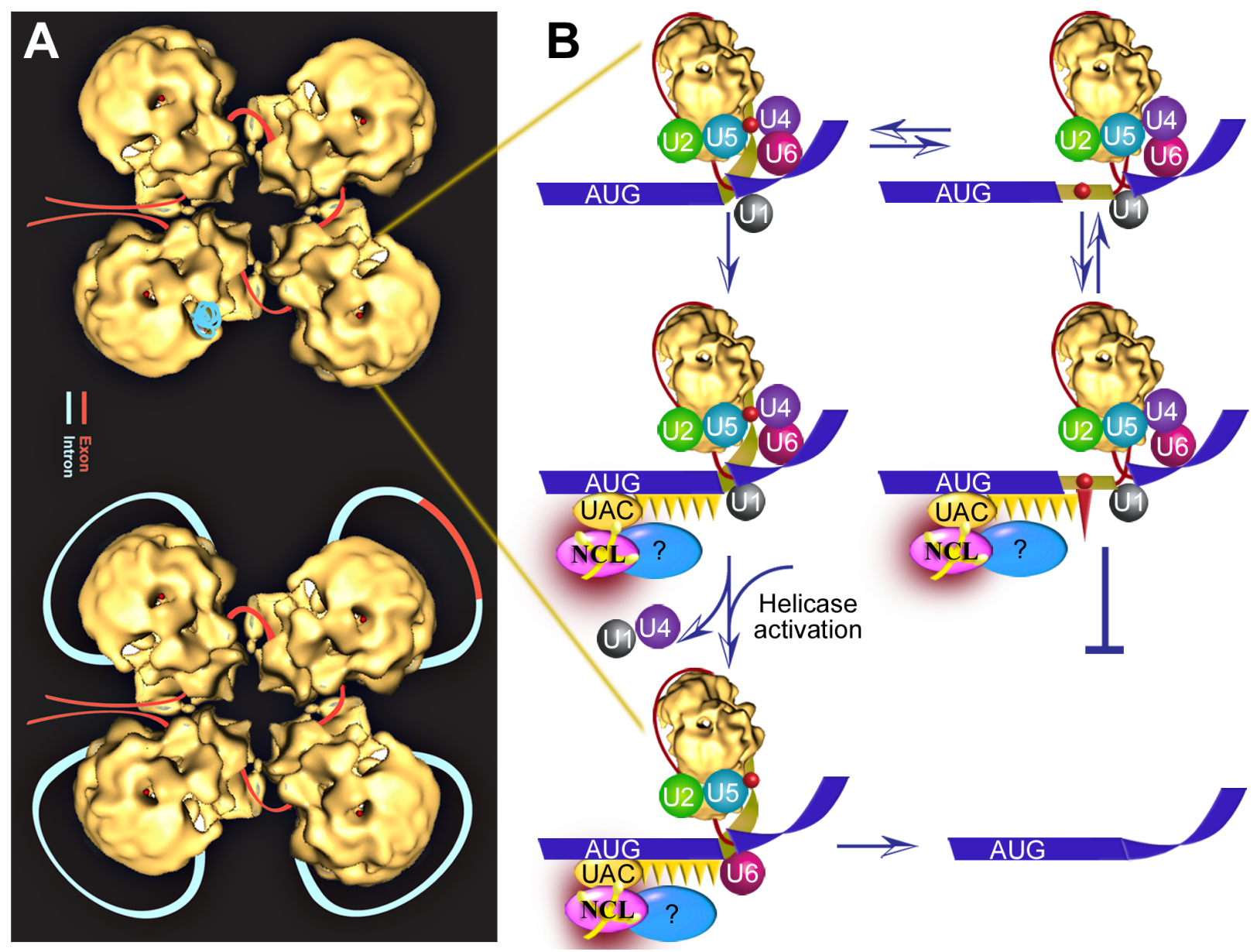

Figure 7. An updated speculative schematic model for the quality control function of SOS. (A) The supraspliceosome model $(50,51,63-65)$. Exon, red; intron, light blue. (Top) The folded pre-mRNA that is not being processed is protected within the cavities of the native spliceosome. (Bottom) When a staining protocol that allows visualization of nucleic acids was used, RNA strands and loops were seen emanating from the supraspliceosomes (66). The RNA kept in the cavity likely unfolded and looped-out under these conditions. In the looped-out scheme an alternative exon is depicted in the upper right corner. (B) Zoom into one spliceosome. Left scheme, splicing at the authentic 5'SS; right scheme, splicing at the latent 5'SS. Blue stripes, exons; red line, intron; yellow narrow stripe, latent exon; red circle, in-frame stop codon; circles, U snRNPs; orange ellipse (UAC), initiator-tRNA; purple ellipse, NCL directly bound with ini-tRNA; and blue ellipse, additional associated components; orange triangles, hypothesized triplet-binding proteins; red triangle, stop-codon-binding protein. Updated from ref. (20). 


\section{Tables}

Table 1. NCL knockdown preferentially activates splicing at latent 5'SS that introduce PTCs.

\begin{tabular}{|c|c|c|c|c|c|c|c|}
\hline \multirow[t]{2}{*}{$\begin{array}{l}\operatorname{A5SS}^{\mathrm{a}} \\
\text { types }\end{array}$} & \multirow[t]{2}{*}{$\begin{array}{l}\text { Introduce } \\
\text { PTC }\end{array}$} & \multicolumn{3}{|c|}{$\begin{array}{l}\text { A5SS activated per sample type, } \\
\text { initial criteria }^{\mathrm{b}}\end{array}$} & \multicolumn{3}{|c|}{$\begin{array}{l}\text { A5SS activated per sample type, stringent } \\
\text { criteria }^{c}\end{array}$} \\
\hline & & NCLsi & CONTsi $^{d}$ & p-value ${ }^{\mathrm{e}}$ & NCLsi & CONTsi $^{\mathrm{d}}$ & p-value ${ }^{\mathrm{e}}$ \\
\hline LSS & Yes & 399 & 82 & $2.3 \times 10^{-11}$ & 187 & 23 & $4.2 \times 10^{-5}$ \\
\hline $\operatorname{adSS}_{3 \mathrm{n}}$ & No & 50 & 50 & N/A & 25 & 16 & N/A \\
\hline $\operatorname{adSS} S_{\mathrm{fs}}$ & Yes & 221 & 52 & $8.5 \times 10^{-9}$ & 112 & 20 & $1.6 \times 10^{-3}$ \\
\hline
\end{tabular}

${ }^{\mathrm{a}}$ Alternative 5 ' splice site. ${ }^{\mathrm{b}}$ Initial activation criteria require read support of at least 4 for the latent junction and increase in relative usage of at least $50 \%$ relative to the control sample in one replicate. For the second replicate, the latent junction is required to be supported by at least one read, while any increase in the relative usage is observed. ${ }^{\mathrm{c}}$ Activation criteria (1.5-fold increase, read support of at least 4$)$ are imposed to both replicates. ${ }^{\mathrm{d}}$ Activation of a $5 \mathrm{SSs}$ is determined by switching NCLsi and CONTsi sample labels and applying the pipeline described in Figure 4C. 'P-value was computed with one-sided Fisher's exact test against the $\operatorname{adSS}_{3 n}$ set. 


\section{Supplementary Figure}

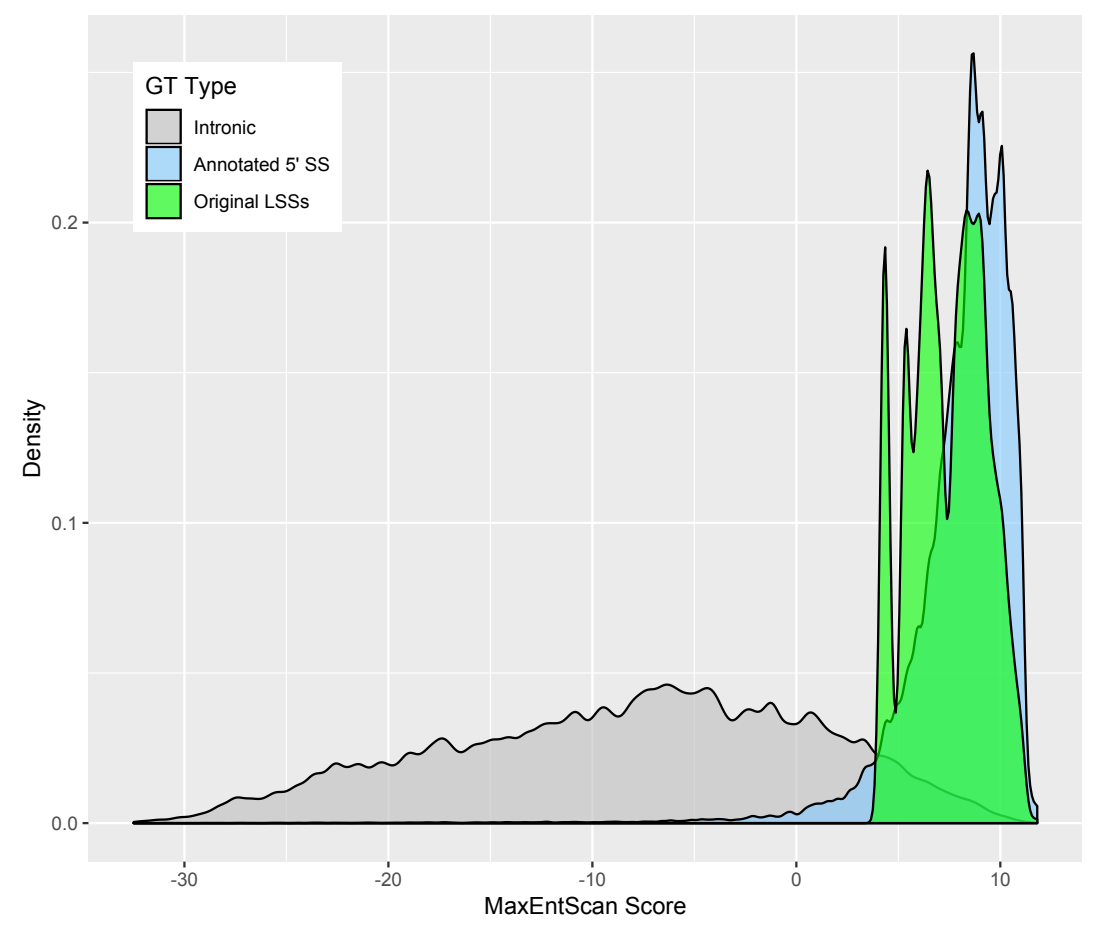

Figure S1. Distribution of MaxEntScan scores for GT dinucleotides in the human genome. The "Intronic" set includes 7762681 GTs located in within-CDS introns. They are limited to those GTs that would extend the upstream exon by at most $1000 \mathrm{nt}$ and would allow a downstream intron of at least $20 \mathrm{nt}$. The "annotated 5' SS" set includes 189037 GTs annotated as donor (5') SSs in NCBI RefSeq, UCSC knownGene, or Gencode (v19) transcripts. Only within-CDS SSs were considered, and SSs with non-canonical consensus dinucleotides were excluded. The "Original LSSs" include 42305 GTs previously identified as LSSs by Nevo et al. (1). Their scores are based on the hg18 assembly of the human genome, whereas the other two sets are based on the data from the hg19 assembly. 


\section{Supplementary Methods}

In vitro Transcription of ini-tRNA. We generated a DNA template using pTRM, a plasmid carrying the WT ini-tRNA gene (2), Q5 DNA polymerase (M0491 NEB), and specific primers in a PCR reaction according to the manufacturer's instructions. In vitro transcription was carried out at $37^{\circ} \mathrm{C}$ for 1 hour in a reaction containing $1.5 \mu \mathrm{g}$ DNA template, $0.5 \mathrm{mM}$ NTPs, $1.5 \mu \mathrm{ci} \alpha{ }^{32} \mathrm{P}$ UTP $(1.8 \mu 1$ BLU007H250UC, Perkin-Elmer), X1 transcription buffer, and 30 units T7 RNA polymerase (Ambion 2718). To generate biotinylated ini-tRNA, we added $0.02 \mathrm{mM}$ biotin UTP to the transcription reaction. To produce cold biotinylated ini-tRNA, we added $0.06 \mathrm{mM}$ cold UTP and $0.02 \mathrm{mM}$ biotinylated UTP to the transcription reaction. After DNase digestion [10 U DNase (Fermentase M610A), $37^{\circ} \mathrm{C}, 20^{\prime}$ ], the RNA was purified using the miRNeasy kit (217004 Qiagen), according to the manufacturer's instructions, and analyzed on a denaturing gel.

Injection of ini-tRNA into the Nuclei of Xenopus Oocytes. We extracted Xenopus oocytes as previously described $(3,4)$. Briefly, Xenopus oocytes were isolated and incubated in NDE96 solution composed of ND96 (96 mM NaCl, $2 \mathrm{mM} \mathrm{KCl,} 1 \mathrm{mM} \mathrm{CaCl}_{2}, 1 \mathrm{mM} \mathrm{MgCl} 2,5 \mathrm{mM}$ Hepes-NaOH, pH 7.5), with the addition of $2.5 \mathrm{mM}$ sodium pyruvate, $100 \mathrm{units} / \mathrm{ml}$ penicillin, and $100 \mu \mathrm{g} / \mathrm{ml}$ streptomycin. A day after oocyte extraction, $10 \mathrm{fmol}(20 \mathrm{nl})$ of ${ }^{32} \mathrm{P}$ ini-tRNA was injected into the oocyte's nucleus (Picospritzer, PLI100; Medical Systems Corp.). After 30 minutes, nuclei were extracted manually using a small needle to pierce the oocyte membrane, and the nuclei were gently squeezed out into GV buffer (5 mM HEPES, 17 $\mathrm{mM} \mathrm{NaCl}, 83 \mathrm{mM} \mathrm{KCl}$ ). Each nucleus was gently washed and transferred to a new plate (5). Radioactivity of nuclei was measured (Perkin-Elmer Tri-Carbs 2900 TR Liquid Scintillation), and only nuclei having over half of the injected ini-tRNA were further assayed. Nuclei were irradiated at $4^{\circ} \mathrm{C}$ with an energy of $0.8 \mathrm{Joule}$ (or 0.5 Joule as indicated) [Ultra-Lum UV Cross-Linker (UVC 515)]. Nuclei were digested by RNase A $\left[1.75 \times 10^{-3}\right.$ RNase A units (Sigma R-6513) per $50 \mu 1$ sample volume] and run on $8.7 \%$ SDS PAGE (1 nucleus/well).

RT-PCR Analysis. RT-PCR was performed on RNA extracted from the NCLsi and CONTsi treated cells as described (1), using the detailed sets of primers (see Primer List). The identity of all PCR products was confirmed by sequencing. Each experiment was repeated at least 3 times. The relative abundance was quantified in view of the intensity of GAPDH used as a control.

\section{Mass Spectrometry Analyses}

Samples Preparation. We incubated 60 Xenopus oocyte nuclei with 120 picomol ini-tRNA (assay samples were prepared with biotinylated UTP and control samples were prepared with non-biotinylated UTP). All samples were affinity purified using $150 \mu \mathrm{C} 1$ streptavidin magnetic beads (as described above) and then resuspended in $100 \mu 110 \mathrm{mM}$ Tris buffer $\mathrm{pH}$ 7.5.

We used in-solution, on-bead, tryptic digestion to prepare samples. We added $8 \mathrm{M}$ urea in $0.1 \mathrm{M}$ Tris ( $\mathrm{pH}$ 7.9) to the beads and incubated them (15 min at RT). Proteins were reduced by incubation with dithiothreitol (5 mM; Sigma; $60 \mathrm{~min}$ at RT) and alkylated with $10 \mathrm{mM}$ iodoacetamide (Sigma) (30 min at $\mathrm{RT}$ in the dark). We diluted the urea solution to $2 \mathrm{M}$ with $50 \mathrm{mM}$ ammonium bicarbonate, added $250 \mathrm{ng}$ trypsin (Promega; Madison, WI, USA), and incubated the reaction overnight at $37^{\circ} \mathrm{C}$. We then added 100 ng trypsin for $4 \mathrm{hr}$ at $37^{\circ} \mathrm{C}$ and stopped the digestion with $1 \%$ trifluroacetic acid (1\% final concentration). Peptides were desalted using Oasis HLB $\mu$ Elution format (Waters, Milford, MA, USA), vacuum dried, and stored at $-80^{\circ} \mathrm{C}$ until further analysis.

Liquid Chromatography. We used ULC/MS grade solvents for all chromatographic steps. Each sample was loaded using split-less nano-Ultra Performance Liquid Chromatography (10 kpsi nanoAcquity; Waters, Milford, MA, USA). The mobile phase was: A) $\mathrm{H}_{2} \mathrm{O}+0.1 \%$ formic acid and B) acetonitrile $+0.1 \%$ formic acid. Desalting of the samples was performed online using a reversed-phase Symmetry C18 trapping column (180 $\mu \mathrm{m}$ internal diameter, $20 \mathrm{~mm}$ length, $5 \mu \mathrm{m}$ particle size; Waters). The peptides were then separated using a T3 HSS nano-column (75 $\mu \mathrm{m}$ internal diameter, $250 \mathrm{~mm}$ length, $1.8 \mu \mathrm{m}$ particle size; Waters) at $0.35 \mu \mathrm{L} / \mathrm{min}$. Peptides were eluted from the column into the mass spectrometer using the following gradient: $4 \%$ to $30 \% \mathrm{~B}$ in $30 \mathrm{~min}, 30 \%$ to $90 \% \mathrm{~B}$ in $10 \mathrm{~min}$, maintained at $90 \%$ for $5 \mathrm{~min}$, and then back to initial conditions.

Mass Spectrometry. We coupled the nanoUPLC online through a nanoESI emitter (10 $\mu \mathrm{m}$ tip; New Objective; Woburn, MA, USA) to a quadrupole orbitrap mass spectrometer (Q Exactive Plus, Thermo 
Scientific) using a FlexIon nanospray apparatus (Proxeon). We acquired data in data dependent acquisition (DDA) mode, using a Top20 method. MS1 resolution was set to 70,000 (at $400 \mathrm{~m} / \mathrm{z}$ ), mass range of 300$1650 \mathrm{~m} / \mathrm{z}$, AGC of $3 \mathrm{e} 6$ and maximum injection time was set to $20 \mathrm{msec}$. MS2 resolution was set to 17,500 , quadrupole isolation $1.7 \mathrm{~m} / \mathrm{z}$, AGC of $1 \mathrm{e} 6$, dynamic exclusion of $60 \mathrm{sec}$ and maximum injection time of $60 \mathrm{msec}$.

Identification of Latent 5'SSs and Alternative Donor SSs. The list of LSSs was obtained with custom Perl software designed to scan the intronic sequences of protein-coding loci corresponding to transcripts from NCBI RefSeq (September 18, 2016), UCSC knownGene (June 30, 2013), and GENCODE v19 (December 5, 2013) annotation tracks. For this purpose we use the hg19/GRCh37 assembly of the human genome. Five criteria were used to define GTs as LSSs: $i$ ) they were located in introns flanked by coding exons, $i$ ) they were no more than 1000 nucleotides downstream of the annotated 5'SS, and accommodating an intron of at least 20 nucleotides with the downstream 3'SS, iii) they scored higher than 0 on the MaxEntScan scale (6), iv) they were not previously annotated as the 5'SS of a separate alternative exon fully contained within the intron investigated, and $v$ ) the translation of the intronic region up to the LSS (i.e., the exon extension) introduced at least one premature termination codon (PTC). For LSSs contained in overlapping transcript isoforms, i.e., those with different reading frames and intron boundaries, analyses were performed separately for each isoform, and only unique LSS instances are reported for LSS counts. With the exception of the requirement for insertion of in-frame STOP codons, the same criteria were used for identifying other alternative donor splice sites $\left(\operatorname{adSS}_{3 \mathrm{n}}, \operatorname{adSS}_{\mathrm{fs}}\right)$. Cases of adSS 3 that introduce new STOP codons that span the exon junction (a total of 805 cases) were considered as adSS $\mathrm{fs}_{\mathrm{f}}$.

Analysis of RNA-Seq Data. For the genome indexing step of the STAR workflow, we included GENCODEv19 gene annotation information and information for all junctions involving LSSs and adSSs. For the alignment step, we set the "--alignSJDBoverhangMin" parameter to 2 to increase alignment sensitivity at junctions of interest, which included both previously annotated junctions and our lists of LSSs and adSSs. Overall, about $84 \%$ of reads from all four samples were uniquely mapped (Table S3) and used for further analyses.

Quantification of Increased LSS Usage. Split reads, i.e. those aligned over an intron-induced gap to exons flanking each side, can usually considered as proof of transcript undergoing the process of splicing. However, when considering cases with increased LSS usage (i.e. fold increase of at least 1.5 in the case over the control sample), we imposed additional conditions to minimize the rate of false positives due to technical artifacts. In addition to a minimum number of 4 reads required, these conditions included: $i$ ) the second replicate was required not to exhibit a decrease in LSS usage (i.e. increase in normalized LSS usage was require to be $\geq 1$ ), $i$ ) at least one split reads was required to support the LSS in the second NCLsi replicate, and iii) both CONTsi replicates were required to have at least 10 split reads that support the canonical junction to indicate that minimal expression can be detected for the canonical junction under normal conditions.

Analysis of Expression Profiles. In building the expression profiles for the exon extension regions we excluded all read pairs for which any part of either read mapped to an intronic region. In this way we minimize the impact of intronic transcriptional noise on evaluating the expression profiles, which is variable across the human genome. Additionally, in evaluating the expression profile, we allowed small deviations from the condition of consistent higher expression level in the NCLsi sample in order to accommodate situation in which reads not originating from the transcripts of interest (e.g. reads generated by intronic transcriptional noise that fully map to exonic regions, reads that originate from alternative isoforms) cannot be readily eliminated. Our pipeline allowed us to eliminate many cases inconsistent with activation of latent splicing at LSS but which showed an increase in LSS usage. Such cases included, but are not limited to, unannotated exons, intronic alternative transcription start sites, or specific amplification of split reads that can happen during construction of sequencing library.

Gene Sets Analysis. Assignment of LSS-containing transcripts to Entrez gene IDs was performed using six cross-reference datasets: "gene2refseq" and "gene2ensembl" from NCBI (ftp://ftp.ncbi.nlm.nih.gov/gene/DATA/, accessed on October 30, 2018), "knownToRefSeq and "knownToEnsembl" from UCSC (http://hgdownload.soe.ucsc.edu/goldenPath/hg19/database/, accessed on 
October 30, 2018), the GENCODE v19 annotation set, as well as a custom reference dataset downloaded through the BioMart interface (https://www.ensembl.org/biomart, accessed on October 30, 2018) containing: Ensembl gene ID, Ensembl transcript ID, NCBI RefSeq and UCSC transcript accession numbers, gene symbol, and NCBI Entrez gene ID. The sets of curate genes were obtained from version 6.2 of MSigDB, which contains 8 gene set collections. Each collection contains between 50 and 5917 individual gene sets (17,810 gene sets in total). Each gene set consists of between 5 and 2940 unique gene identifiers, provided as gene symbols and Entrez gene IDs. To correctly evaluate enrichment in biological properties for the genes with activated LSSs, all genes included in the analysis (i.e. genes from the MSigDB gene sets, as well as genes in the background set) were required to have evidence of $i$ ) at least one LSS with a MaxEntScan score above 0.05 and ii) support of at least 10 split reads in both CONTsi samples for the canonical junction encompassing the suitable LSSs in order to match the genes that could be detected as having activated LSSs.

\section{Primer List}

Specific primers for ini-tRNA generation were designed:

Sense primer: 5'- TAATACGACTCACTATAAGCAG - 3', and antisense primer: 5'- TGGTAGCAGAGGATGGTTTC- 3'

elongator ini-tRNA template primer: 5'TGGTGCCCCGTGTGAGGATCGAACTCACGACCTTCAGATTATGAGACTGACGCGCTACCTA CTGCGCTAACGAGGCTATAGTGAGTCGTATTA-3

T7 primer: 5'-TAATACGACTCACTATA-3'

Primer sets for the RT-PCR validation experiments:

\section{RAF1}

Sense primer 5' - TCTGGCATGTTGAGGGCTTTG -3'

Antisense primer 5'- ACTTTGGTGCTACAGTGCTCA - 3'

Annealing temp $57^{\circ} \mathrm{C}$

\section{ANK2}

Sense primer 5'- GAGCTTGTGTCCTCTACATG - 3'

Antisense primer 5'- TCCAGCTGTCCCCATTCTCA - 3'

Annealing temp $56^{\circ} \mathrm{C}$

\section{VRK2}

Sense primer 5'- GCAAAGCAAGCTACTTCCAT - 3'

Antisense primer 5'- AATTCAGTCAGACCAGATCC - 3'

Annealing temp $51.5^{\circ} \mathrm{C}$

\section{KIAA0169}

Sense primer 5'- TGTGGCACAGCCCCTATTCCA - 3'

Antisense primer 5'- AGACCAAAGGTTCCCAAGGTG - 3'

Annealing temp $58^{\circ} \mathrm{C}$

\section{COPA}

Sense primer 5'- CAGAAGCGGTAACATATCAGG - 3'

Antisense primer 5' - CGTGTTTGGCTAGTAGTGCT - 3'

Annealing temp $51^{\circ} \mathrm{C}$

\section{LARS}

Sense primer 5'- TTGCGGCAGAAAATAGACCTA - 3'

Antisense primer 5'- GTCACACAGAGCCACAACAC - 3

Annealing temp $55^{\circ} \mathrm{C}$

\section{HTT}

Sense primer 5'- CAGGTCGTCCTTGTGTTAGGA - 3'

Antisense primer 5 - CTTGCATGGTGGAGAGACGA - 3'

Annealing temp $57^{\circ} \mathrm{C}$ 


\section{TCERG1}

Sense primer 5'- GTCAGAAAACAATCTTTGGGGG - 3'

Antisense primer 5' - TGTGCAACTCCTTCTCCCAC - 3'

Annealing temp $55^{\circ} \mathrm{C}$

\section{HEATR1}

Sense primer 5' - CAATTGAGAAATGGTGCTAGTC - 3'

Antisense primer 5' - TGATGAATGATGGAGACGACCA - 3'

Annealing temp $51.5^{\circ} \mathrm{C}$

\section{GAPDH}

Sense primer 5'- CCAGCCGAGCCACATCGCTC - 3'

Antisense primer 5' - TGAGCCCCAGCCTTCTCCAT - 3'

Annealing temp $60.0^{\circ} \mathrm{C}$

\section{References}

1. Y. Nevo et al., Genome-wide activation of latent donor splice sites in stress and disease. Nucleic Acids Res. 40, 10980-10994 (2012).

2. T. V. Pestova et al., Molecular mechanisms of translation initiation in eukaryotes. Proc. Natl. Acad. Sci. USA 98, 7029-7036 (2001).

3. Y. Ben-Chaim, O. Tour, N. Dascal, I. Parnas, H. Parnas, The M2 muscarinic G-protein-coupled receptor is voltage-sensitive. J. Biol. Chem. 278, 22482-22491 (2003).

4. L. Ohana, O. Barchad, I. Parnas, H. Parnas, The metabotropic glutamate G-protein-coupled receptors mGluR3 and mGluR1a are voltage-sensitive. J. Biol. Chem. 281, 24204-24215 (2006).

5. E. H. Birkenmeier, D. D. Brown, E. Jordan, A nuclear extract of Xenopus laevis oocytes that accurately transcribes 5S RNA genes. Cell 15, 1077-1086 (1978).

6. G. Yeo, C. B. Burge, Maximum entropy modeling of short sequence motifs with applications to RNA splicing signals. J. Comput. Biol. 11, 377-394 (2004).

\section{Supplementary Tables}

Table S1. List of peptides identified by mass spectrometry.

Table S2. List of latent splice sites (LSSs) identified in the human genome.

Table S3. Summary of reads obtained through the RNA-Seq experiment and mapped to each of the four samples.

Table S4. List of genes with differential expression in NCLsi compared to CONTsi samples.

Table S5. List of LSSs activated in NCLsi samples upon NCL knockdown.

Table S6. Gene sets over-represented among the genes with activated LSSs.

Table S7. List of LSSs activated in CONTsi samples.

Table S8. List of adSS $\mathrm{Sn}_{3 \mathrm{n}}$ splice sites identified in the human genome.

Table S9. List of adSS ${ }_{3 n}$ activated in NCLsi samples upon NCL knockdown.

Table S10. List of adSS $\mathrm{Sn}_{3 \mathrm{n}}$ activated in CONTsi samples.

Table S11. List of adSS $\mathrm{f}_{\mathrm{fs}}$ splice sites identified in the human genome.

Table S12. List of adSS $\mathrm{fs}_{\mathrm{fs}}$ activated in NCLsi samples upon NCL knockdown.

Table S13. List of adSS $\mathrm{fs}_{\mathrm{f}}$ activated in CONTsi samples. 